\title{
Symptomatic and Disease-Modifying Effects of GABAA Receptor Positive Allosteric Modulation in a Mouse Model of Chronic Stress
}

Ashley Bernardo ${ }^{1}$, Philippe Lee ${ }^{2}$, Michael Marcotte ${ }^{1}$, Yeunus Mian ${ }^{3}$, Zubair A. Khan ${ }^{3}$, Dishary Sharmin ${ }^{3}$, James M. Cook ${ }^{3}$, Etienne Sibille $e^{1,2,4}$ and Thomas D. Prevot ${ }^{1,4^{*}}$

${ }^{1}$ Campbell Family Mental Health Research Institute of CAMH, Toronto, Canada

${ }^{2}$ Department of Pharmacology and Toxicology, University of Toronto, Toronto, Canada

${ }^{3}$ Department of Chemistry and Biochemistry, University of Wisconsin-Milwaukee, Milwaukee, USA

${ }^{4}$ Department of Psychiatry, University of Toronto, Toronto, Canada

*Corresponding Author

Thomas D. Prevot, Ph.D., CAMH, 250 College Street, room 131, Toronto, ON M5T 1R8, Canada

Tel: 416-535-8501, ext. 33785; E-mail: thomas.prevot@camh.ca 


\begin{abstract}
:
Chronic stress is a major risk factor for developing depressive disorders and animal models of stress recapitulate behavioral, cellular and molecular changes that are observed in human depression. Individuals exposed to chronic stress, or patients with MDD experience mood and cognitive dysfunctions. This is in part due to neuronal shrinkage in brain regions involved in several cognitive functions such as the prefrontal cortex (PFC) and the hippocampus (HPC). Also in the context of depression and chronic stress, expression levels and function of the main inhibitory neurotransmitter GABA are reduced. Thus far, drugs targeting this GABA deficit have failed to produce beneficial effects due to broad activity at various GABA receptor subunits, including the $\alpha 1$-subunit, resulting in broad side effects. However, refined and selective activity at the $\alpha 2 / 3 / 5$-subunit is hypothesized to exert beneficial effect, devoid of side effects.
\end{abstract}

Here, we show that GL-II-73 and GL-I-54 exert positive allosteric modulation at the $\alpha 5$, and $\alpha 2 / 3 / 5$ contianing GABAA receptors respectively, and that they are effective both independently and in combination. Using unpredictable chronic mild stress (UCMS) experiments in male and female C57BL/6 mice ( $n=12$ per group), we showed that acute and chronic administration of a GL-II-73/GL-I-54 racemic mixture (termed "GL-RM") reduced anxiety-like phenotypes and reversed a working memory deficit in UCMS exposed mice. Brains from animals receiving chronic treatment were collected and stained using a Golgi staining technique. Using stereological approaches, neuronal morphology was reconstructed and dendritic length, spine count and spine density were assessed in pyramidal neurons of the PFC and hippocampus. Chronic GL-RM rescued spine density depletions caused by UCMS at apical and basal dendrites (PFC and CA1). Interestingly, spine densities in both brain regions were correlated to cognitive performance, confirming ameliorative benefits of GL-RM.

Together, results support the value of selectively targeting GABAA receptors, excluding the $\alpha 1$-subunit, to overcome chronic stress-induced mood symptoms and cognitive deficits, as well as detriments in neuronal morphology. This study confirm results that were observed in old mice, using a $\alpha 5$-selective positive allosteric modulator, and reinforce the concept that the $\alpha 2 / 3 / 5$-containing GABAA receptor are suitable targets for the treatment of stress-induced disorders. 


\section{Introduction}

Major Depressive Disorder (MDD) is the leading cause of disability in the world, affecting 322 million people worldwide [1]. Patients suffering from MDD experience a plethora of symptoms spanning from low mood, anxiety, anhedonia to cognitive dysfunctions, not always treated with current therapies [2-4]. Underlying pathologies of MDD encompass dysfunctions in the monoaminergic system [5], the GABAergic system [6], neuroendocrinology dysfunctions [7], and reduced neuronal complexity [8]. While the pathology is very complex in nature, current interventions rely only on reversing the monoaminergic imbalance, which shows limited efficacy on mood symptoms, no efficacy on cognitive symptoms, and do not act on other underlying pathologies encountered in MDD. Importantly, even if mood symptoms are alleviated, cognitive dysfunctions remain [9]. It has been proposed that mood and cognitive symptoms are interconnected, and that persisting cognitive symptoms in remission can predict mood symptoms relapse [10-13]. Therefore, there is a critical need for a better understanding of underlying pathologies in the context of MDD that can help develop novel therapeutic options with an impact on both mood and cognitive symptoms. Developing new therapeutic avenues for psychiatric disorders that target the underlying pathologies and contribute to alleviation of the symptoms is extraordinarily challenging, but also features a fantastic potential to help millions of patients worldwide.

In depressed patients, it has been shown that the connectivity between neurons is decreased [14], potentially contributing to the severity of the disease [15]. Indeed, post-mortem research has demonstrated that number of synapses, and expression of synaptic-function-related genes $[14,16,17]$ are reduced in the dorsolateral prefrontal cortex (PFC) of patients suffering from MDD. Similar findings are reported in chronic stress-related disorders [15, 18], including in animal models [19-21]. Chronic stress induces various changes on dendritic length, spine density and connectivity in various brain regions of the limbic system, such as the PFC, the hippocampus (HPC) or the amygdala [22]. In the PFC and the HPC, chronic stress induces reduction of dendritic length and spine density, while it causes 
increase of same in various subnuclei of the amygdala [22]. Because of the involvement of the PFC and the HPC on cognitive functions, the impact of chronic stress on neuronal morphology in these two regions is thought to partially contribute to the cognitive deficits while increase in amygdala connectivity is suggested to participate in increased anxiety phenotype observed in chronic stress-related disorders. Structural changes at the macroscale level are now acknowledged as part of the disease, but to our knowledge, only ketamine has shown efficacy at increasing spine density and dendritic complexity in mice, with combined antidepressant properties [23]. While the mechanisms involved in the neurotrophic effects of ketamine remain unknown, its fast-acting antidepressant properties have been suggested to be mediated by activity on GABAergic neurons [24], main support of brain inhibitory neurotransmission contributing to the brain excitation/inhibition balance.

As mentioned before, human studies showed that patients with MDD exhibit impaired excitation/inhibition balance, caused in part by altered GABAergic (inhibitory) functions [6]. Indeed, live and post-mortem studies showed reduced GABA levels in cerebrospinal fluid [25], plasma [26-31] and in brain regions such as the occipital cortex [32-36] in treated and untreated depressed patients [37, 38], in the PFC [39] and anterior cingulate cortex (ACC) [32, 39-41] of depressed patients. However, there is no treatment option that aims at reducing this imbalance in MDD patients. GABA signal through GABAA and $G A B A B$ receptors, the former being ionotropic channels and the latter being metabotropic receptors coupled to G-proteins. GABAA receptors (GABAA-Rs) are pentameric receptors, formed by a combination of 19 subunit subtypes (taken from $\alpha, \beta, \gamma, \delta, \varepsilon, \theta, \pi$ and $\rho$ subunits), most often forming a channel with $2 \alpha, 2 \beta$ and $1 \gamma$ subunit [42]. Drugs acting on the GABAA -Rs exist (such as benzodiazepines; BZs), but their use is limited because of broad activity at various receptor subunits, ultimately causing various side effects and risk of dementia disorders [43, 44]. Selective targeting of GABAA-Rs, specifically by potentiating $\alpha 2, \alpha 3$, and $\alpha 5$ GABAA R's while limiting potentiation of $\alpha 1$, is hypothesized to improve the therapeutic potential of a compound by reducing the broad spectrum of side effects and sedation 
that occur with current therapeutics such as BZ's $[43,45]$. Studies demonstrated that targeting $\alpha 2 / 3$ GABAA-Rs has beneficial effect on mood and anxiety $[46,47]$. Other studies have found the $\alpha 5$-subunit of GABAA-Rs to be linked with cognitive performance $[48,49]$. Anatomically, $\alpha 5$ containing receptors are most abundant within the HPC and MPFC of mice [50], both regions being highly involved in cognitive processes [51]. Based on these observations, our group and others already demonstrated that targeting the $\alpha 5$-GABAA-Rs with a positive allosteric modulator has beneficial effects on cognitive [52] and mood functions in mouse models. More specifically, we demonstrated beneficial effects of targeting $\alpha 5-$ GABAA-Rs on working memory in the context of chronic stress [49], and we confirm such effects in the contexts of normal aging [53].

The use of animal models for the development of novel therapies for psychiatric disorders is debated yet essential despite translational limitations of their use [22, 54]. Multiple models have been developed and used over the years [55] but the unpredictable chronic mild stress (UCMS) is the most widely used model of chronic stress-related disorders [56]. This model is strongly associated with anhedonia behavior, anxiety-like behaviors and cognitive deficits in rodents [55], and overcomes habituation to a stressor [57]. It is also known to disrupt GABA signaling, contributing to mood and cognitive symptoms $[58,59]$. Previous studies, including from our group showed that chronic stress in rodents, similarly to what is observed in humans [60-63], decreases GABA release, alters GABA-related gene expression and disrupts receptor function [59, 64-68]. From these observations, targeting the GABAergic system, and more specifically the signaling pathway involving GABAA-Rs involved in the regulation of mood and cognition, appears as a valid strategy to develop novel antidepressant $[67,69]$.

Novel strategies compared to what is already available need to be explored, and here we focus on the GABAergic system as an underestimated avenue for the treatment of depressive pathophysiology. We hypothesized that treatment with combined treatment acting on the $\alpha 5-$ GABAA-Rs and the $\alpha 2 / 3$ 
GABAA-Rs using PAMs, will contribute to alleviating symptoms associated with chronic stress exposure in mouse. We also tested the hypothesis that chronic treatment extends its effects on symptoms to underlying pathologies, by reversing neuronal atrophy observed after chronic stress. To do this, we used animal models of chronic stress, with acute and chronic administration of $\alpha$-PAMs to investigate their symptomatic and neurotrophic effects. 


\section{Materials and Methods}

Details on the listed methods are provided in the supplementary material.

\section{Electrophysiology}

Electrophysiological recordings were performed, using standard methods in the field [70, 71], on HEK293T cell line transfected with full-length cDNAs for the human GABAA receptor subtypes ( $\alpha 1 \beta 3 \gamma 2$, $\alpha 2 \beta 3 \gamma 2, \alpha 3 \beta 3 \gamma 2, \alpha 4 \beta 3 \gamma 2$ or $\alpha 5 \beta 3 \gamma 2$ ). Holding potential was $-70 \mathrm{mV}$. Current recordings after first application of the test articles alone were taken to detect potential agonist effects (Supplementary Figure 1.) followed by exposure of each compound in the presence of GABA (Figure 1 and Supplementary Figure 2). GL-II-73 or GL-I-54 was applied to cells at a range of concentrations $(0.033 \mu \mathrm{M}$, $0.1 \mu \mathrm{M}, 0.33 \mu \mathrm{M}, 1 \mu \mathrm{M}, 3.33 \mu \mathrm{M}, 10 \mu \mathrm{M}, 33.33 \mu \mathrm{M})$ in presence of $5 \mu \mathrm{M}$ GABA.

\section{Liver microsomal Assay}

Briefly, the compounds were tested for the metabolic stability in C57BL/6 Mouse liver microsomes at a $2 \mu \mathrm{M}$ concentration and $0.5 \mathrm{mg} / \mathrm{mL}$ of Matrix concentration, as in Di et al.[72]. NADPH solution and compounds were added to assay plates and then the plate was quenched. Microsomes/S9 were quickly thawed and added to the assay to start reaction and the time was recorded. For T0, duplicate aliquots were transferred to a quench plate and mixed. This was repeated at 30,60, and 120 . Sample plate was sealed and put into a refrigerated LC/MS/MS autosampler for assessment. Calculations for calculated intrinsic hepatic clearance used $45 \mathrm{mg}$ microsomes/g liver and $87.5 \mathrm{~g}$ liver/ $/ \mathrm{kg}$ body weight for mouse.

\section{Animals}

All animal cohorts were obtained from Jackson Laboratories (Stock\# 000664; Bar Harbor, MA, USA) consisting of 8-week old C57BL/6 mice with 50\% female mice in each cohort. Four separate cohorts were used for the purpose of this study. The first cohort was composed of 42 mice to assess the effect of GL-I-54 on working memory. The second cohort was composed of 18 mice to test GL-I-54 effects in the elevated plus maze. The third and fourth cohorts were composed of 48 mice each ( $N=12$ per group, $50 \%$ females) with $50 \%$ of each cohort subjected to unpredictable chronic mild stress (UCMS), and assessing the acute or chronic effects of GL-RM treatment (combined GL-I-54 and GL-II-73), respectively. All animals were maintained on a 12 hour light/dark cycle (7:00 ON, 19:00 OFF), given access to food and water ad libitum (unless otherwise required by testing) and housed individually. Mice were handled for 5 minutes on 3 consecutive days to reduce stress and anxiety during experimenter/mouse interactions 
and behavioral tests. For all studies, animals were assigned a blind number and divided into groups with a 50\% sex-ratio. All animal work was completed in accordance with Ontario Animals for Research Act (RSO 1990, Chapter A.22), the guidelines of the Canadian Council on Animal Care (CCAC) approved by the Animal Care Committee at CAMH.

\section{Drug Preparation and administration}

Preliminary behavioral data for GL-I-54 used an acute intraperitoneal injection (IP) at $10 \mathrm{mg} / \mathrm{kg}$. GL-I-54 was dissolved in vehicle solution ( $85 \%$ distilled $\mathrm{H}_{2} \mathrm{O}, 14 \%$ propylene glycol (Sigma Aldrich) and $1 \%$ Tween 80 (Sigma Aldrich)) and injected 30 minutes prior to running behavioral testing.

For the 2 UCMS studies, a racemic preparation containing 50\% of GL-II-73 and 50\% of GL-I-54 was synthesized. Here, we will refer to this racemic mix as GL-RM. For acute use, GL-RM was dissolved in vehicle solution, as described above. Acute administration of GL-RM used IP as the route of administration with a dose of $10 \mathrm{mg} / \mathrm{kg}$. Solutions were made daily for acute administration and administered 30 minutes prior to behavioral testing. For the acute drug treatment in the PhenoTyper, mice were acutely injected with saline or GL-RM $(10 \mathrm{mg} / \mathrm{kg}$ ) before 20:00hr (Depicted by black arrow in Figure 2F). The chronic administration study used the per oral (PO) route with $266 \mathrm{~mL} / \mathrm{kg}$ as the administration volume amounting to a $30 \mathrm{mg} / \mathrm{kg}$ dose. For this preparation, GL-RM was dissolved in tap water overnight at room temperature and prepared every other day. The GL-RM was then made available to the animals through their drinking water.

\section{Stress Paradigms}

\section{Chronic Restraint Stress (CRS)}

Mice were placed in a $50 \mathrm{~mL}$ Falcon ${ }^{\text {TM }}$ Tube with a hole at the conical tip and a hole in the lid which allowed for breathing and physical space. The tube was returned to the home cage and left for one hour. This was repeated twice daily (separated by at least 1 hour) for 1 week.

\section{Unpredictable Chronic Mild Stress (UCMS)}

The UCMS paradigm used a variety of mild stressors (1-2 applied daily) that were randomly performed over 6 weeks ( 8 weeks considering the 2 weeks of behavioral testing). During the testing phase, 1 stressor per day was applied after testing. Animals subjected to UCMS were housed in a separate room 
without environmental enrichment to exacerbate the effects of other stressors. (Full description in supplementary methods).

\section{Coat state}

Coat state of each animal was monitored weekly to determine the effect of UCMS and the progressive reduction in self-care, as described in Nollet et al. [73]. Coat state was scored for seven anatomical areas of each animal's coat on a 0-1 rating scale. $0=$ well-groomed with a smooth coat, $1=$ soiled coat or with bald patches.

\section{Behavioral Tests}

Prior to behavioral testing, animals were moved to the testing room and allowed to habituate to the room and lighting conditions for 30 minutes. Prior to placing the animal in any apparatus, the apparatus was cleaned with $70 \%$ ethanol, and left for $2 \mathrm{~min}$ to dry in order to remove the odor from the previously tested animal.

\section{Elevated Plus Maze (EPM)}

The EPM is commonly used in preclinical rodent models to assess anxiety-like behaviors and was used as per standard method[74]. Briefly, mice are placed in a cross-shaped maze raised at $55 \mathrm{~cm}$ from the floor, with 2 open arms facing each other, and 2 closed arms facing each other for $10 \mathrm{~min}$. Exploration of each mouse was recorded using a digital camera mounted on the ceiling. The videos were analyzed using Ethovision XT14 software. Specific parameters considered were: \% time spent in the open arms, calculated using ([(total time spent in open arms)/(total time spent in open arms + total time spent in closed arms)]*100) and the number of open arm entries.

Open Field (OF)

The OF test is designed to test anxiety-like behavior in rodents through their ambulatory movements in an enclosed arena. The open field test was run in a $43 \times 43 \times 50 \mathrm{~cm}$ square chamber with grey floor and dark grey walls. One mouse was placed in a single field and recorded for 10 minutes by the same system used in the EPM. Time spent and number of entries into the center were used as measures of anxietylike beahviors.

Novelty Suppressed Feeding (NSF) 
NSF is often used to detect stress induced anxiety-like behavior by measuring a rodent's reluctance to eat in an unfamiliar environment. The NSF test chamber is a $62 \times 31 \times 40 \mathrm{~cm}$ Plexiglas container with clear floors and walls. Mice were weighed and food deprived for 16 hours prior to testing. The following day, animal weights were assessed ensuring an $8-12 \%$ weight loss. A familiar kind of food pellet was placed $\sim 8 \mathrm{~cm}$ from the wall of the chamber on the side closest to the experimenter and the mouse was placed on the opposite side of the chamber. A timer was started immediately and the latency to approach and consume the food pellet was recorded by an experimenter. After the test was completed mice were returned to their home cage where latency to bite/feed on the food pellet was tested to control for appetite drive or food deprivation-induced weight loss. A maximum of 12 minutes is allotted for testing in the novel environment and 6 minutes in the animal's home environment.

PhenoTyper $^{\text {TM }}(P T)$ Test

The assay was performed as per methods described in Prevot et al[74]. The PhenoTyper box consists of a home cage setting, with food hopper, water bottle and a shelter. The ceiling of the box is equipped with an infrared camera allowing videotracking with Ethovision, and a white light that can be turned on as needed for experimental purposes. From $11 \mathrm{pm}$ to $12 \mathrm{am}$ (at night), a white light shines above the food zone and represents a stressful stimulus for the mouse. The design of this assay includes characterization of the activity of the mouse before, during and after the stressful stimulus.

Anxiety-like behavior can be reproducibly quantified by investigating time spent in food and shelter zones and residual avoidance can be calculated based on each zone. Residual avoidance (RA) is an index calculated as a function of the control group response to the light challenge and subsequent 5 hours. A positive RA value indicates avoidance of the lit zone and is calculated using the following equations based on the time spent in food zone or time spent the shelter zone. Food Zone: [1-( $\Sigma$ Time (12am5am)-Time (11pm-12am))/Average control group ( $\Sigma$ Time (12am-5am)-Time (11pm-12am))]*100, where "Time" is time spent in food zone. Shelter Zone: [( $\Sigma$ Time (12am-5am)-Time (11pm$12 \mathrm{am})) /$ Average control group ( $\Sigma$ Time (12am-5am)-Time (11pm-12am))-1]*100, where "Time" is time spent in shelter zone. Chronically stressed mice have a predictable avoidance behavioral pattern after an aversive light stimulus that is detectable in the PhenoTyper ${ }^{\mathrm{TM}}$ [74].

\section{Sucrose Consumption (SC)}

Sucrose consumption can be used as a measure of anhedonia-like behavior. Mice were habituated to a $1 \%$ sucrose drinking solution for 48 hours followed by fluid deprivation overnight ( 16 hours). To test 
sucrose consumption, the weight of each $1 \%$ sucrose solution tube is recorded and then given to individually housed mice, where they have free access to the solution. After 1 hour, the tubes are removed and tube weights are recorded again. Similar design is used for water consumption the following days. \% Sucrose consumption was then calculated using the following equation: \% Sucrose Consumption $=[($ Sucrose consumed $) /$ sucrose consumed + water consumed $)]^{*} 100$.

Forced Swim Test (FST)

FST is used to detect depressive-like behavior. For this test mice were placed in a 4 liter beaker (1 mouse /beaker) containing 3 liters of water (23]C-25]C) for a total of 6 minutes. A digital camera filmed the mice swimming from the side. After 6 minutes, mice were removed from the water, dried with a towel and warmed under a heat lamp for 5 minutes. Videos were analyzed using EthoVision XT 14 software after acquisition. Total time immobile (immobility defined as the minimum movement to keep the head above water) was analyzed for minutes 0-4 and reported.

\section{Y Maze}

The $Y$ maze is a spatial working memory paradigm looking at spontaneous alternation[49, 53] and adapted from the T maze paradigm described by Faucher et al[75]. After habituation to the maze, mice are tested for spontaneous alternation, with a $90 \mathrm{sec}$ inter-trial interval (ITI), all trial starting from the "start box". For each trial, the mouse can choose between the left or right arm, and one a choice is made, the mouse stays in the chosen arm for $30 \mathrm{sec}$, before returning to the start box for $90 \mathrm{sec}$ (ITI). This is repeated over 7 trials. Between each trial the maze is wiped down lightly with $70 \%$ ethanol to remove olfactory cues. Also, an $8^{\text {th }}$ trial is performed with a short ITI $(10 \mathrm{sec})$ to test the animal's motivation to alternate. Animals that do not alternate on the $8^{\text {th }}$ trial show reduced motivation and therefore are excluded form analysis as their results are biased by lack of motivation and not cognitive performance. The mean alternation rate was calculated using the following formula and expressed as a percent: Mean alternation $\%=[($ Number of alternations/number of trials $) * 100]$.

\section{Tissue Collection}

Mice were euthanized by cervical dislocation 24 hours after performing the final behavioral test. Four brains were collected from 3 groups (vehicle treated non-UCMS ("Control"), vehicle treated UCMS and GL-RM treated UCMS). Once extracted from the skull the brains were used for Golgi-Cox staining, performed by NeuroDigiTech. Each brain was immersed in Golgi-Cox staining solution provided by 
NeuroDigiTech LLC and assigned a unique identifier. Brains were then shipped to NeuroDigiTech for full analysis.

\section{Golgi Staining Analysis}

Neuronal morphology was assessed using Golgi Staining. The analysis was performed by NeuroDigiTech (San Francisco CA., USA). After staining, brains were sectioned on a cryostat ( $100 \mu \mathrm{m}$ thickness), and mounted on glass slides. Basal and apical dendrites of pyramidal cells were identified in layers II/III of the PFC and in the CA1 of the hippocampus and determined to be the regions of interest (ROls). For quantification, NeuroLucida v10 (Microbrightfield, VT) software was used on a Dell PC computer and controlled a Zeiss Axioplan 2 image microscope with Optronics MicroFire CCD camera (1600x1200). Neurons ( $n=6$ per animal) were analyzed for their dendritic length, spine number and spine density, as per the methods described in Wu et al.[76]. For spine sampling, only those orthogonal to the dendritic shaft were well characterized and included as those above or below could not be adequately distinguished. Raw data was extrapolated and quantified using NeuroExplorer (Microbrightfield, VT).

\section{Design}

After habituation in the facility, mice are tested in the PhenoTyper for a baseline assessment of their response to the light challenge. After a baseline characterization of the animal behavior, mice are split into 2 groups, control or UCMS. During the UCMS protocol, each week, mice were tested in the PT boxes, tested for sucrose consumption and their coat state and weight were measured. Timelines for acute and chronic studies are depicted in Figures $2 \mathrm{~A}$ and $\mathbf{3 A}$ respectively.

\section{Statistics}

\section{Z-Scores Calculation}

Z-scores for emotionality were calculated to give an overall representation of anxiety-like behavioral phenotypes and depressive-like behavioral phenotypes. Z scores for each animal were calculated for each parameter of the behavioral tests included in each respective score using the following formula: $\mathrm{Z}$ score $=[($ Individual raw data-Average control raw data) $/($ Standard deviation control raw data) $]$ or $\mathbf{Z}$ score $=[($ Individual raw data-Average control raw data)/-(Standard deviation control raw data) $]$ dependent on the original test's raw values (higher values for higher anxiety, or lower values for higher anxiety, respectively). Z-scores for all parameters where then averaged for each animal in a single Zemotionality, including z-scores from EPM (Time spent in open arms and open arm entries), NSF 
(Latency to approach and latency to bite), PhenoTyper (week 6 food zone residual avoidance and shelter zone residual avoidance), coat state, FST (time immobile) and sucrose consumption at week 6.

\section{Statistical Testing}

Electrophysiology data of GL-II-73 and GL-I-54 and their activity at GABA receptor subunits was compared to $100 \%$ using T-test. Then, overall activity was analyzed using two-way ANOVA. Independent variables were considered as concentration and receptor subunit type. Bonferroni post hoc testing was used to identify differences at specific subunits using GraphPad Prism 5 software. Preliminary GL-I-54 EPM behavioral testing was analyzed using t-test and GL-II-73 behavioral testing was analyzed using one-way ANOVA with Fisher's lest significant difference post-hoc test (PLSD). UCMS studies that involved weekly assessment were analyzed using repeated measures two-way ANOVA (coat state, weight gain, PhentoTyper time spent in specific zones and residual avoidance scores). Standard two-way ANOVA was used to evaluate all other behavioral outcomes from the different tests (EPM, OF, NSF, SC, FST and $\mathrm{Y}$ maze) considering both UCMS and treatment as independent variables. Fishers PLSD tests were used for post hoc analysis. StatView software was used for behavioral testing statistical analysis and sex effects. Golgi staining employed repeated measures one-way ANOVA with Tukeys post hoc tests. All correlation analysis was conducted using GraphPad Prism 5 software and used Spearman ranks correlation between morphological features and behavioral outcomes. For all comparisons statistical significance is considered $\mathrm{p}<0.05$ and all data are presented as the mean \pm SEM. 


\section{$\underline{\text { Results }}$}

GL-II-73 and GL-I-54 exhibit potentiation at $\alpha 5-, \alpha 3-, \alpha 2-$ and $\alpha 1-G A B A A$ receptors and show metabolic stability

First, we investigated the electrophysiolgical profiles of the two compounds independently to determine their activity at human recombinant $\alpha 1-5$ GABAA-Rs. In absence of GABA in the media, neither GL-II-73 nor GL-I-54 induced potentiation at any of the receptor, confirming their role as allosteric modulator and not agonist (Supplementary Fig. 1).

When tested in the allosteric mode (in presence of GABA), GL-II-73 significantly increased potentiation

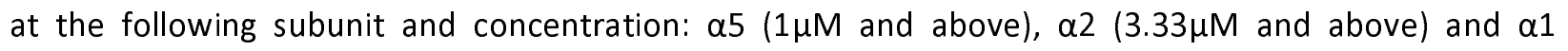
$(33.33 \mu \mathrm{M})$. (Supplementary Results and Supplementary Fig. 2A). Then, a repeated measure ANOVA was used to detect preferential selectivity of one subunit type over the others (Fig. 1A). Considering all concentrations, repeated measure ANOVA revealed an effect of subunit type $\left(F_{(4 ; 105)}=26.22 ; p<0.0001\right)$, concentration $\left(F_{(6 ; 105)}=14.96 ; p<0.0001\right)$, and an interaction $\left(F_{(24 ; 105)}=2.463 ; p=0.0009\right)$. Post hoc testing found $\alpha 5$ was significantly potentiated over $\alpha 1$ at $0.3 \mu \mathrm{M}(\mathrm{p}<0.05), 3.33 \mu \mathrm{M}(\mathrm{p}<0.001), 10 \mu \mathrm{M}(\mathrm{p}<0.001)$ and $33 \mu \mathrm{M}$ ( $\mathrm{p}<0.001$ ). Selectivity for $\alpha 5$ over $\alpha 2, \alpha 3$ and $\alpha 4$ was also detected at several concentrations $(\alpha 2: 3.3 \mu \mathrm{M}(\mathrm{p}<0.01), 10 \mu \mathrm{M}$ (p<0.001); $\alpha 3: 1 \mu \mathrm{M}(\mathrm{p}<0.05), 3.3 \mu \mathrm{M}(\mathrm{p}<0.01), 10 \mu \mathrm{M}(\mathrm{p}<0.001) ; \alpha 4: 3.33 \mu \mathrm{M}$ $(p<0.001), 10 \mu \mathrm{M}(\mathrm{p}<0.001), 33 \mu \mathrm{M}(\mathrm{p}<0.001)) . \alpha 2$ preference was only found at the highest concentration $(33 \mu \mathrm{M})$ between $\alpha 1(p<0.05)$ and $\alpha 4(p<0.001)$. Full post hoc testing results can be found in Supplementary Table 1A. Together, data show GL-II-73 is a selective $\alpha 5$ PAM at concentrations below $10 \mu \mathrm{M}$.

Regarding GL-I-54, tested in allosteric mode, it significantly increased potentiation at $\alpha 2(0.03 \mu \mathrm{M}$ and

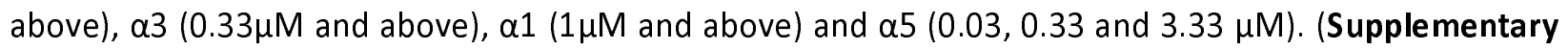
Results and Supplementary Fig. 2B). Then, a repeated measures ANOVA, used to identify $\alpha 1-5$ preferences, detected subunit differences $\left(F_{(4 ; 105)}=81.19 ; p<0.0001\right)$, concentration dependent effects $\left(F_{(6 ; 105)}=19.4 ; p<0.0001\right)$ and an interaction between subunit and concentration by $G L-I-54\left(F_{(24 ; 105)}=5.201\right.$; p<0.0001; Fig. 1B). Post hoc testing identified preferential activity at $\alpha 2$ over $\alpha 1, \alpha 3, \alpha 4$ and $\alpha 5$ at several concentrations, followed by $\alpha 3$ preference over $\alpha 1, \alpha 4$ and $\alpha 5$. $\alpha 5$ and $\alpha 1$ were potentiated over $\alpha 4$ while no preference was detected between $\alpha 5$ and $\alpha 1$. Due to the complex potentiation profile of GL-I-54 at high concentrations, only concentrations within physiologically relevant ranges for in vivo use were considered $(0.1 \mu \mathrm{M}-1 \mu \mathrm{M})$. Results found, $\alpha 2$ was significantly targeted over $\alpha 1(0.3 \mu \mathrm{M} ; \mathrm{p}<0.01)$ 
and $\alpha 4(0.3 \mu \mathrm{M}(\mathrm{p}<0.001), 1 \mu \mathrm{M}(\mathrm{p}<0.001)), \alpha 3$ was selectively targeted over $\alpha 1$ and $\alpha 4$ at all physiologically relevant concentrations $(0.1 \mu \mathrm{M}-1 \mu \mathrm{M})$ and $1 \mu \mathrm{M}$ over $\alpha 5$. Preference for $\alpha 5$ was detected over $\alpha 4$ at $1 \mu \mathrm{M}(\mathrm{p}<0.01)$ and $\alpha 1$ preference was not significant over any other subunit type (full post hoc analysis can be found in Supplementary Table 1B). These results together demonstrate that GL-I-54 has preferential PAM activity for $\alpha 2$ and $\alpha 3$ followed by $\alpha 5$ within the physiologically utilized concentration range.

Using a liver microsomal assay, metabolism and stability of each compound was assessed. GL-II-73 was found to be stable in the C57BL6 mouse with a half-life of 256 minutes. After 2 hours, $75.6 \%$ of GL-II-73 was remaining and the calculated intrinsic hepatic clearance was $21.3 \mathrm{~mL} / \mathrm{min} / \mathrm{kg}$. In the $\mathrm{C} 57 \mathrm{BL} / 6 \mathrm{mouse}$, GL-I-54 was also stable and found to have a half-life of 198 minutes, with $70 \%$ remaining after 2 hours. The calculated intrinsic hepatic clearance for GL-I-54 was determined to be $27.6 \mathrm{~mL} / \mathrm{min} / \mathrm{kg}$.

\section{In vivo anxiolytic and pro-cognitive effects of GL-II-73 and GL-I-54}

Anxiolytic properties and benefits on working memory of GL-II-73 have previously been published by our group [49]. Separate cohorts of mice were tested in the elevated plus maze and in the $Y$ maze to assess anxiolytic effect and efficacy on working memory of GL-I-54, respectively. In the EPM, an unpaired t-test found trends towards significance for $\%$ time spent in the open arms $\left(t_{(10)}=2.004 ; p=0.07 ;\right.$ Fig. 1C) and number of open arm entries $\left(t_{(10)}=1.754 ; p=0.1\right.$; Fig. 1D). The trends suggested more time spent and more entries into open arms were made by animals receiving GL-I-54. No sex differences were identified for either EPM parameter (\% time $\left(t_{(10)}=-0.142 ; p=0.889\right)$, entries $\left(t_{(10)}=0.043 ; p=0.9667\right)$ ). Beneficial effects on working memory were found with GL-I-54, in the $Y$ maze test (Fig. 1E). An overall group effect was found $\left(F_{(4,29)}=6.682 ; p=0.0006\right)$ and post hoc analysis determined CRS induced working memory deficits. GL-I-54 was found to have a significant effect on $\mathrm{Y}$ maze alternation rate. Post hoc analysis revealed significant differences between CRS-Vehicle vs CRS-10mg $/ \mathrm{kg}$ GL-I-54 ( $p=0.0223$ ) and no significant difference between Control-Vehicle vs CRS-10mg/kg GL-I-54 ( $p=0.1342$ ) showing full cognitive recovery at $10 \mathrm{mg} / \mathrm{kg}$.

Results from this point forward used a racemic mixture of GL-I-54 and GL-II-73 (referred herein as GLRM), due to their enantiomeric nature, the combined preferred subunit profiles and their similar anxiolytic effect and efficacy on working memory.

Acute administration of GL-RM has antidepressant, anxiolytic effects and pro-cognitive effects in mice subjected to UCMS 
The effects of acute administration of GL-RM on symptoms induced by chronic stress were studied in the UCMS model as described in Fig. 2A. Coat state and weight gain were measured every week for all animals throughout the study (Supplementary Results and Supplementary Fig. 3). Briefly, UCMS induced deterioration of the coat state that was not reversed by acute treatment. UCMS did not alter in weight gain.

\section{Anxiolytic effects of GL-RM after acute administration}

Animals were tested in the EPM and ANOVA found UCMS $\left(F_{(1 ; 42)}=0.0791 ; p=0.379\right)$ and treatment alone $\left(F_{(1 ; 42)}=1.377 ; p=0.2472\right)$ had no effect on \% time spent in the open arms but the interaction between UCMS and treatment was approaching significance $\left(F_{(1 ; 42)}=3.675 ; p=0.062\right)$ (Fig.2B). No significant effect of sex was identified $\left(F_{(1 ; 38)}=0.508 ; p=4802\right)$. The number of open arm entries was not affected by UCMS $\left(F_{(1 ; 42)}=0.29 ; p=0.5929\right)$ but was significantly increased by $G L-R M$ as found by ANOVA $\left(F_{(1 ; 42)}=7.317\right.$; $p=0.0098$; Fig. 2C). No significant interaction was found between UCMS and treatment $\left(F_{(1 ; 42)}=1.972\right.$; $p=0.1684)$. Sex effects were also not significant $\left(F_{(1 ; 38)}=2.68 ; p=0.11\right)$.

Two-way ANOVA on latency to approach in the NSF test found a trend level effect of UCMS $\left(F_{(1 ; 41)}=2.927\right.$; $p=0.0947)$ and no effect of $G L-R M\left(F_{(1 ; 37)}=0.003 ; p=0.9538\right)$ but did identify a significant interaction for UCMS*treatment $\left(\mathrm{F}_{(1 ; 41)}=5.477 ; \mathrm{p}=0.0242\right)$. Post hoc testing found UCMS exposed GL-RM treated animals exhibited reduced approach latencies compared to control GL-RM treated animals $(p=0.0284$ ) and GLRM reduced approach latency in animals exposed to UCMS compared to those treated with vehicle $(p=0.0113)$ (Fig.2D). Sex remained non-significant $\left(F_{(1 ; 37)}=1.912 ; p=0.175\right)$. Latency to bite found no significant effect of UCMS $\left(F_{(1 ; 41)}=0.671 ; p=0.4175\right)$, drug $\left(F_{(1 ; 41)}=0.588 ; p=0.4475\right)$ or interaction between UCMS*treatment $\left(F_{(1 ; 41)}=0.981 ; p=0.3277\right)$ (Fig. 2E). Sex effects also did not affect latency to bite $\left(F_{(1 ; 35)}=0.163 ; p=0.6886\right)$.

The Phenotyper test was used to assess anxiety-like behavior on a weekly basis. Baseline activity in the boxes were recorded prior to UCMS to ensure to artificial differences pre-existing between groups prior to UCMS or treatment (Supplementary Fig. 4). Once the UCMS began, overall time spent in the food zone (Supplementary Results and Supplementary Fig.5) and shelter zone (Fig.2F and Supplementary Fig.6) were analyzed. ANOVA performed on the time spent in the shelter on week 6 showed an effect of $\operatorname{UCMS~}\left(F_{(1 ; 44)}=37.715 ; p=<0.001\right)$ but no effect of treatment $\left(F_{(1 ; 44)}=1.519 ; p=0.2243\right)$ or interaction $\left(F_{(1 ; 44)}=0.202 ; p=0.6557\right)$. Sex had a significant effect $\left(F_{(1 ; 40)}=16.061 ; p=0.0003\right)$ and Bonferroni post hoc testing identified significantly increased time spent in the shelter zone by males compared to females. 
Residual avoidance for the shelter zone was calculated at week 6 and a significant effect of UCMS was confirmed using a two-way ANOVA $\left(\mathrm{F}_{(1 ; 44)}=23.964 ; \mathrm{p}<0.0001\right)$. No effect of treatment was found $\left(F_{(1 ; 44)}=0.182 ; p=0.6714\right)$ and there remained no interaction $\left(F_{(1 ; 44)}=0.499 ; p=0.4835\right)$ at week 6 (Fig. 2G). An effect of sex was found $\left(F_{(1 ; 46)}=16.046 ; p=0.0002\right)$ with females showing less residual avoidance than males.

\section{GL-RM has potential to improve depressive-like phenotypes after acute administration}

Depressive-like and anhedonia-like behaviors were evaluated using the forced swim test and sucrose consumption paradigm, respectively. At week 6 , sucrose preference was significantly reduced by UCMS $\left(F_{(1 ; 42)}=4.230 ; p=0.0460\right)$ and unaffected by $G L-R M \quad\left(F_{(1 ; 42)}=0.256 ; p=0.6714\right)$ (Fig. 2H). No interaction between UCMS*treatment was found $\left(F_{(1 ; 42)}=1.971 ; p=0.48535\right)$. No effect of stress was found $\left(F_{(1 ; 38)}=1.423 ; p=0.2403\right)$. The forced swim test was conducted on week 8. ANOVA found time immobile was significantly affected by UCMS $\left(F_{(1 ; 42)}=11.060 ; p=0.0018\right)$ and treatment $\left(F_{(1 ; 42)}=12.313 ; p=0.001\right)$ with no interaction between the two factors $\left(F_{(1 ; 42)}=0.164 ; p=0.6879\right)$. Post hoc testing identified these effects were due to significantly longer time spent immobile by vehicle treated UCMS animals than vehicle treated controls ( $p=0.0341$ ), and GL-RM reduced immobility time in UCMS animals compared to vehicle treated UCMS animals $(p=0.0094)$. No effect of sex was found $\left(F_{(1 ; 38)}=1.492 ; p=0.2295\right)$ (Fig. 2I). Using Zscoring to evaluate Z-emotionality, two-way ANOVA found an overall effect of UCMS $\left(F_{(1 ; 40)}=45.937 ; p<0.0001\right)$, no significant effect of treatment $\left(F_{(1 ; 40)}=2.491 ; p=0.1224\right)$ and no interaction $\left(F_{(1 ; 40)}=0.324 ; p=0.5723\right)$ (Fig. 2J). Sex effects were also not significant $\left(F_{(1 ; 36)}=0.652 ; p=4248\right)$. This describe the potential role of GL-RM at reversing part of the behavioral changes caused by chronic stress.

\section{Spatial working memory is improved by acute treatment of GL-RM}

Spatial working memory was investigated using the Y-maze task after acute administration of GL-RM. Two-way ANOVA analysis on the alternation rate showed a significant effect of UCMS $\left(F_{(1 ; 18)}=11.62\right.$; $p=0.003)$, no effect of treatment $\left(F_{(1 ; 18)}=1.748 ; p=0.2027\right)$ and a significant interaction for UCMS*treatment $\left(\mathrm{F}_{(1 ; 18)}=16.85 ; \mathrm{p}=0.0007\right)$. Post hoc analysis revealed UCMS significantly decreased the alternation rate of animals receiving vehicle $(p=0.0013)$; and GL-RM administration increased the alternation rate in animals subjected to UCMS ( $p=0.0022$; Fig. $2 K$ ). No effect of sex was found $\left(F_{(1 ; 14)}=0.034 ; p=0.8567\right)$. Together, this describes the capability of GL-RM to overcome the cognitive deficit induced by UCMS. 


\section{Chronic treatment with GL-RM reduces emotionality and rescues spatial working memory}

Based on beneficial effects found with acute administration of GL-RM, chronic administration was subsequently investigated in the same UCMS model. It is important to note that chronic administration began on week 3 of UCMS (Fig. 3A). Coat state and weight gain were again measured weekly and confirmed what was seen in the previous study (Supplementary Results and Supplementary Fig.8), i.e. increased coat deterioration with UCMS.

\section{Potential anxiolytic effects of chronic treatment with GL-RM}

In the EPM, two-way ANOVA performed on percent time spent in the open arms found no effect of $\operatorname{UCMS}\left(F_{(1 ; 44)}=0.564 ; p=0.4565\right)$, treatment $\left(F_{(1 ; 44)}=0.515 ; p=0.515\right)$ or interaction $\left(F_{(1 ; 44)}=0.502 ; p=0.4822\right)$ (Fig. 3B). No sex effect was found $\left(F_{(1 ; 40)}=1.003 ; p=0.3225\right)$. Number of entries into open arms similarly found no effect of UCMS $\left(F_{(1 ; 44)}=0.59 ; p=0.8088\right)$, treatment $\left(F_{(1 ; 44)}=2.133 ; p=0.1513\right)$ or interaction $\left(F_{(1 ; 44)}=0.948 ; p=0.3356\right)$ (Fig. 3C). Sex also did not have an effect $\left(F_{(1 ; 40)}=2.227 ; p=0.1434\right)$. In the open field, ANOVA analysis also did not detect an effect of UCMS $\left(F_{(1 ; 44)}=0.055 ; p=0.8160\right)$, treatment $\left(F_{(1 ; 44)}=0.706 ; p=0.4053\right)$ or an interaction $\left(F_{(1 ; 44)}=1.046 ; p=0.3121\right)$ on time spent in the inner zone (Fig. 3D). No sex effects were found $\left(F_{(1 ; 40)}=0.122 ; p=0.7288\right.$. The number of inner zone entries remained unaffected by UCMS $\left(\mathrm{F}_{(1 ; 44)}=0.472 ; \mathrm{p}=0.4956\right)$, treatment with $\mathrm{GL}-\mathrm{RM}$ had a trend level affect approaching significance $\left(\mathrm{F}_{(1 ; 44)}=3.487 ; \mathrm{p}=0.0685\right)$ and no interaction between UCMS*treatment was found $\left(F_{(1 ; 44)}=0.777 ; p=0.3828\right)$ (Fig. 3E). Sex effects remained non-significant $\left(F_{(1 ; 40)}=3.287 ; p=0.0773\right)$. Mice were tested in the novelty-suppressed feeding test on week 6 . ANOVA performed on the latency to approach the pellet did not show an effect of UCMS $\left(F_{(1 ; 44)}=0.496 ; p=0.4851\right)$, treatment $\left(F_{(1 ; 44)}=0.410 p=0.5255\right)$ or interaction $\left(F_{(1 ; 44)}=0.542 ; p=0.542\right)$ (Fig. 3F). ANOVA performed on the latency to bite detected a significant effect of UCMS $\left(F_{(1 ; 44)}=6.871 ; p=0.0120\right)$ and of drug treatment $\left(F_{(1 ; 44)}=6.723 ; p=0.0129\right)$ with no significant interaction $\left(F_{(1 ; 44)}=1.256 ; p=0.2685\right)$. Post hoc analysis found the difference likely due to treatment differences in control animals $(p=0.0537)$ and the stress condition of GL-RM treated animals ( $p=0.0216$ ) (Fig. 3G). No effect of sex was found $\left(F_{(1 ; 40)}=1.043 ; p=0.3134\right)$.

In the Phenotyper test, when GL-RM was onboard (weeks 3-6), a repeated measures ANOVA on the residual avoidance scores for the shelter zone found a significant effect of UCMS $\left(F_{(1 ; 44)}=60.082\right.$; $p=<0.0001)$, a trend level effect of treatment $\left(F_{(1 ; 44)}=2.819 ; p=0.09\right)$ and no significant interaction $\left(F_{(1 ; 44)}=2.377 ; p=0.1303 ;\right.$ Fig. 3H $)$. 


\section{Chronic GL-RM has beneficial influence on emotionality scores}

In the FST, two-way ANOVA performed on the time spent immobile did not detect an effect of UCMS $\left(F_{(1 ; 44)}=0.025 ; p=0.8759\right)$, treatment $\left(F_{(1 ; 44)}=0.034 ; p=0.8542\right)$ and no significant interaction $\left(F_{(1 ; 44)}=1.206\right.$; $\mathrm{p}=0.2781$ ) (Fig. 3I). In the sucrose consumption test, two-way ANOVA performed on sucrose consumption identified no significant effect of $U C M S\left(F_{(1 ; 44)}=1.606 ; p=0.2117\right)$ but did detect a significant effect of $G L-R M\left(F_{(1 ; 44)}=6.294 ; p=0.0159\right)$. No significant interaction was detected between UCMS and treatment $\left(\mathrm{F}_{(1 ; 44)}=0.67 ; \mathrm{p}=0.7968\right) \quad \mathrm{GL}-\mathrm{RM}$ (Fig. 3J). Z-score analysis considering Z-emotionality determined that UCMS had a significant heightening effect on z-emotionality $\left(F_{(1 ; 44)}=6.219 ; p=0.0165\right)$ and treatment also significantly affected z-emotionality $\left(F_{(1 ; 44)}=3.965 ; p=0.05\right)$ with no significant interaction being detected $\left(F_{(1 ; 44)}=0.34 ; p=0.56\right)$. Post hoc testing did not significantly distinguish specific group differences responsible for these overall effects (Fig. 3K). Sex did not have an effect $\left(F_{(1 ; 40)}=0.048\right.$; $\mathrm{p}=0.8275)$.

\section{Remedial cognitive effects are observed with chronic GL-RM administration}

$\mathrm{Y}$ maze results identified similar cognitive benefits to acute administration findings. Two-way ANOVA found UCMS had a detrimental effect on alternation rate $\left(F_{(1 ; 37)}=6.330 ; p=0.0163\right)$ while treatment with GL-RM ameliorated these effects $\left(F_{(1 ; 37)}=9.258 ; p=0.0043\right)$. UCMS*treatment were found to have a significant interaction $\left(\mathrm{F}_{(1 ; 37)}=4.755 ; \mathrm{p}=0.0356\right)$. Post hoc testing identified these effects were due to significant differences between control vehicle animals and vehicle treated animals subjected to UCMS $(p=0.005)$. Additionally, animals subjected to UCMS that were treated with GL-RM performed significantly better than those treated with vehicle ( $p=0.0006)$ (Fig. 3L). No sex differences were found $\left(F_{(1 ; 33)}=0.165 ; p=0.6874\right)$.

\section{Neuronal morphology deficits induced by UCMS are prevented by GL-RM}

Using a Golgi staining approach combined with neuron tracing, we quantified morphology changes in apical and basal dendrites of pyramidal neurons in the PFC (Fig. 4 A-C). Analyses of dendritic length and spine count in the basal and apical segment of pyramidal neurons from the PFC are presented in Supplementary Results and Supplementary Fig.13. Spine densities for basal and apical segments of PFC neurons are shown in Fig. 4D and showed that UCMS significantly reduces spine density in both segment while GL-RM reverse such deficits. Indeed, repeated measures ANOVA revealed group differences at basal dendrites $\left(F_{(2 ; 69)}=25.28 ; p<0.0001\right)$. Tukey's post hoc tests showed a decrease in spine density caused by UCMS (Control vs UCMS $p<0.0001$ ) that is partially recovered by GL-RM treatment (UCMS vs 
UCMS+GL-RM $p<0.0001$ and Control vs UCMS+GL-RM $p=0.0128)$. The same deficit was found at apical dendrites $\left(\mathrm{F}_{(2 ; 69)}=26.01 ; \mathrm{p}<0.0001\right)$ with post hoc testing revealing a similar impact of UCMS (Control vs UCMS p 0.0001). A more complete recovery was made by GL-RM at apical dendrites as GL-RM treated animals did not differ from the control group significantly (UCMS vs UCMS+ GL-RM $p=<0.0001$, Control vs UCMS+ GL-RM p=0.6713).

Similar analyses were performed on principle neurons of the CA1 (Supplementary Results, Supplementary Fig. 14 and 15). Repeated measures ANOVA performed on the spine density in the CA1, shown in Fig. 4E, found group differences for both a basal $\left(F_{(2 ; 69)}=34.56 ; p<0.0001\right)$ and apical dendrites $\left(F_{(2 ; 69)}=19.73 ; p<0.0001\right)$. Post hoc testing revealed UCMS consistently reduced spine density compared to control animals (basal and apical $p<0.0001$ ), while GL-RM administered to UCMS animals significantly increased spine density compared to UCMS vehicle treated mice in basal $(p<0.001)$ and in apical $(p<0.01)$ dendrites. At both segments this was a partial recovery as post hoc analysis found spine density in UCMS+GL-RM animals were still lower than controls (basal $p<0.001$, apical $p<0.01$ ).

\section{Synaptic spine architecture in the PFC and CA1 are related to spatial working memory and emotionality}

Spine density in the PFC and CA1 were correlated to spatial working memory performance of the $Y$ maze. In both regions, positive correlations were found at apical and basal segments, suggesting that lower alternation rates are associated with lower spine densities within the PFC as shown in Fig. 5A and Fig. 5B (basal spearman $r=0.7616 ; p=0.004$, apical spearman $r=0.6217 ; p=0.0309$ ). A similar positive correlation was found for spine density and y maze performance when analyzing the CA1 (basal spearman $r=0.6615 ; p=0.0191$, apical spearman $r=0.7496$; $p=0.005$; Fig. 5C and Fig. 5D). Correlations for dendritic length and spine count in both regions with $y$ maze performance can be found in Supplementary Results and Supplementary Fig. 16.

The correlations between Z-emotionality and each morphological outcome were explored within the PFC and CA1. In the PFC, Spearman correlations detected no significant correlation between Zemotionality and any morphological parameter analyzed (spine count: basal $p=0.8970$, apical $p=0.9484$; dendritic length: basal $p=0.9484$, apical $p=0.3079$; spine density: basal $p=0.9304$, apical $p=0.4399$ ) (Full results in Supplementary Table 2). In the CA1 of the hippocampus, a negative correlation was found between Z-emotionality and basal dendritic length (spearman $r=-0.7622 ; p=0.004$ ) (Fig. 5E). At apical dendrites Z-emotionality was also negatively correlated with dendritic length (spearman $r=-0.7552$; 
bioRxiv preprint doi: https://doi.org/10.1101/2021.03.22.436517; this version posted March 23, 2021. The copyright holder for this preprint (which was not certified by peer review) is the author/funder. All rights reserved. No reuse allowed without permission.

$p=0.0045$ ), suggesting increased dendritic length was correlated with lower z-emotionality scores (Fig. 5F). No correlations were found between Z-emotionality and spine count (basal $p=0.0754$, apical $p=0.1309$ ) or spine density (basal $p=0.1055$, apical $p=0.1942$; Supplementary Fig. 17). 


\section{Discussion}

This study is based on the observation that patients suffering from depression experience various symptoms including low mood, anxiety, anhedonia and cognitive dysfunctions, which are not treated with current therapies [2-4]. Part of this lack of efficacy can be attributed to the fact that current therapies do not target underlying pathologies, such as reduced inhibition and reduced neuronal complexity [8]. Here, we proposed to investigate the effects of a racemic mix GL-RM, made of two compounds enantiomer of each other, with activity at $\alpha 5$-GABAA-Rs (GL-II-73) and at $\alpha 3 / 2 / 5-G A B A A-R s$ (GL-I-54), supposedly exerting beneficial effects on mood, cognition and neuronal morphology in mouse models [46-49, 77, 78]. We demonstrate here that GL-RM has beneficial effect on mood and working memory in animal models of chronic stress. Additionally, we demonstrate that chronic treatment with GL-RM reverses neuronal atrophy in the PFC and the CA1 of mice subjected to chronic stress, and that such morphological features correlate with measure of emotionality (anxiety-like and depressive-like behaviors) as well as working memory scores.

Data obtained from this study showed that, while structurally identical, the isomerization of the two compounds tested seems to play a critical role in their positive allosteric role at the GABAA receptors. Indeed, GL-II-73 showed highly preferential activity at the $\alpha 5-G A B A A-R s$, with minor activity at $\alpha 2 / 3-G A B A A-R s$, while GL-I-54 seems to have a broader activity at $\alpha 3 / 2 / 5 / 1$, in this order of preference. This difference in electrophysiology profile can be due to different interaction of the two compounds in the binding pocket, with the conformation of GL-I-54 being more prone to interact and activate $\alpha 1 / 2 / 3$-containing GABAA receptors, compared to GL-II-73 which seems to be more specific to $\alpha 5-G A B A A-R s$. Electrophysiological profile of GL-II-73 was previously described in Prevot et al[49], and here we confirm this profile and extend it to more concentration points.

Previous studies from our group showed that GL-II-73 has anxiolytic, antidepressant effects and efficacy on working memory $[49,78]$, and here we showed that these effects are also elicited with GL-I54. We believe that this shared efficacies between the 2 enantiomers, despite apparent differences in electrophysiological profiles, is due to their activity at $\alpha 2 / 3 / 5-G A B A A-R s$, even if their amplitude of potentiation is different. As previously mentioned, $\alpha 2-$ and $\alpha 3-G A B A A-R s$, strongly present in the amygdala $[50,79,80]$ and the dentate gyrus and CA1-CA3 of the HPC $[50,81,82]$ are commonly linked to regulation of anxious and depressive phenotypes [83, 84]. Also, manipulations of the $\alpha 2 / 3-G A B A A-R s$ with agonists have shown that these receptor subtypes mediate anxiolytic effects [83, 85]. Here we confirm that targeting these receptors with GL-I-54 alone or GL-RM exert similar effects. In addition, 
more and more studies suggest a critical role of the $\alpha 5$-GABAA-Rs in the regulation of cognitive processes $[48,49,78,86]$. The $\alpha 5-G A B A A-R s$ are highly expressed in the mPFC and the HPC [50], both regions highly involved in cognitive processes, further suggesting their implication in the regulation of signal input in cognitive tasks [86].

It has been hypothesized that fine tuning of the GABAergic system is a safer way to elicit beneficial effects [87], and here, we believe that mild activity at the $\alpha 2 / 3-G A B A A-R s$ from GL-II-73, and mild activity at the $\alpha 5-$ GABAARs from GL-I-54 are enough to exhibit beneficial effects in all symptom dimensions tested. One potential caveat of the broad activity profile of GL-I-54, is its potentiation effect at the $\alpha 1-$ GABAA-Rs, which contributes to sedative and addictive properties of classical BZs [88]. While other studies from our group showed that GL-I-54 causes sedation at higher doses (data not shown), lowering the dose of this particular compound is important to avoid side-effects. In such case, the use of lower doses of GL-I-54 and its combination with its enantiomer GL-II-73 which already has reduced-tono side-effect would provide beneficial combination of potentiation and symptoms relief, and provides a rationale for testing racemic mixture of the two compounds.

The use of racemic compounds has brought attention within the treatment of depression and anxiety due to variable effects with various isoforms of a molecule [89]. Majority of therapeutics are marketed as pure compounds and are sometimes considered superior to racemic mixtures. Arguments for their superiority include reduced side effects, reduced dose requirements, increased therapeutic window, increased receptor selectivity and less drug interactions [90, 91]. While different selectivity profiles can burden some racemic mixtures, GL-RM harnessed the similar, yet different selectivity profiles of GL-I-54 and GL-II-73 to specifically target a range of GABAA-Rs known to play a role in anxiety, depression $(\alpha 2, \alpha 3)$ and cognition $(\alpha 5)$ while still limiting those involved in sedation and other unwanted effects $(\alpha 1)$.

Behavioral observations support this at face value as sedative effects were not observed during any testing regimen at the dose tested. Overall, this potentiation profile adds a clinical advantage to using the racemic mixture over either pure enantiomer alone. Fluoxetine and Citalopram are clinically used anti-depressants that are currently marketed racemic compounds [89, 92, 93]. Racemic compounds used clinically often demonstrate similar pharmacological profiles between enantiomers with only minor differences. Enantiomers in Fluoxetine share many common pharmacologic traits with the exception that R-Fluoxetine has a 4 times greater clearance rate than S-Fluoxetine [94]. Investigation of GL-II-73 and GL-I-54 found differing selectivity profiles for GABAA-Rs, however half-life data and 
clearance rate data were found to be relatively similar ( $0.3 x$ higher clearance of GL-I-54), suggesting that they would be no major pharmacology differences between the two enantiomers.

To determine the potential benefice of using GL-RM for its efficacy on mood and cognitive symptoms, we worked with animal models of chronic stress. As in many animal models, replication between laboratories varies due to different stressor durations and the variety of stressors being applied. The protocol in this study used several of the most commonly reported stressors [57]. Despite this, variation in anxiety phenotypes was found even after weeks of UCMS exposure and has also been reported by others $[74,95]$. In both acute and chronic studies the EPM did not find a significant effect of UCMS, limiting the anxiolytic conclusion of GL-RM with regards to this test. The anxiety phenotype induced by UCMS and evaluated using NSF was only observed in the acute study where GL-RM did remedy anxiety-like behavior. Anxiolytic effects of GL-II-73 have been demonstrated previously [49], and we showed here that GL-I-54 also exhibited anxiolytic properties. It would be expected that GL-RM should exhibit anxiolytic properties, but the effect was not clearly identified via the EPM or the NSF. Interestingly, the Phenotyper test and the measure of residual avoidance, provided additional insight on the anxiolytic properties of GL-RM. Indeed, residual avoidance is used as a measure of anxiety-like behavior, referring to context anxiety [74]. In both studies, UCMS significantly increased the residual avoidance, which was significantly reversed by chronic GL-RM treatment. This discrepancy between the EPM/NSF results and the Phenotyper results also highlight the potential bias that can emerge when using hands-on behavioral assays versus automatized approaches. With advances in technologies, more and more assays rely on automation, or experimenter-free approaches in order to reduce bias linked to experimenter handling or indirect influence on behavioral outcomes [96].

Using the UCMS model, depressive-like symptoms (assessed with coat state and the FST) were less variable than anxiety-like and became present as early as week 1 of UCMS, exemplified by coat state analysis. Depressive-like phenotypes, combined to overall impact on anxiety-like phenotypes were used to build an emotionality z-score. In both studies, the emotionality z-score showed a significant effect of UCMS confirming the impact of the UCMS procedure on the behavioral outcomes. Comparing the two treatment regiments, chronic administration was able to overcome the increased emotionality of UCMS animals supporting better efficacy with longer administration, as expected for antidepressant therapy.

Chronic stress is a risk factor for developing depression and exert a major impact on GABAergic functions [60-63]. Indeed, GABA interneurons, specifically SST interneurons are highly vulnerable to stress $[97,98]$. Loss of SST cells function results in a loss of inhibition onto pyramidal neurons and 
ultimately disrupts controlled firing. The lack of inhibitory SST inputs reduces both temporal and spatial signal to noise ratios at pyramidal neurons [99] which leads to a lack of synchronicity between the PFC and HPC and dysfunctional information processing within cortical and hippocampal structures [100]. Loss of controlled pyramidal excitation, in addition to the physical loss of dendritic spines within mPFC and HPC after chronic mild stress, leads to cognitive impairment that can manifest as impaired spatial working memory as seen in this study. This study confirmed UCMS exposure impaired spatial working memory using the $\mathrm{Y}$ maze alternation task. Our findings are consistent with other UCMS paradigms reporting cognitive deficits in working memory [101-103]. These deficits were remedied by both acute and chronic administration of GL-RM. Improvement of working memory was the most robust effect, which could be due to the high selectivity of GL-II-73 for a5-GABAA-Rs.

Neural correlates of stress such as reduced spine density, spine count and impaired dendritic arborization, are linked to emotionality, depression and cognitive performance [98]. In this study, UCMS exposure had a severe impact on dendritic spines, reducing spine density in both the MPFC and CA1 of the HPC which can impact connectivity, and synchronicity required for cognitive processing. Conversely, GL-RM was able to partially remedy these effects in both brain regions. Correlation analyses also suggested that higher spine density was associated with higher performances on spatial working memory. Subunit-specific neurotrophic mechanisms of GL-RM were not determined, however it is known that the CA1 region of the hippocampus highly expresses $\alpha 5, \alpha 3$ and $\alpha 2$ GABAA-Rs in humans and mice $[104,105]$. Previously, $\alpha 5$ and $\alpha 2$ GABAA-Rs have been implicated in dendritic outgrowth and spine maturation as well as synapse formation [106-108] thus their modulation by GL-RM may be responsible for spine density restoration at apical and basal dendrites. These results are consistent with chronic administration of GL-II-73 alone remedying age-related spine loss within apical segment of pyramidal neurons, reported in Prevot et al.[53]. In this study, the authors found neurotrophic effects through $\alpha 5$ modulation confined to apical dendrites. Here, we observed neurotrophic effects extending to basal and apical segments. This difference may be present because $\alpha 5-G A B A A-R s$ are primarily located at apical dendrites $[105,109,110]$ and GL-II-73 alone is preferentially selective for $\alpha 5$. Using GLRM we may have extended the neurotrophic effect to basal dendrites through modulation of $\alpha 2$ and $\alpha 3$. While we show in this study that spine counts are improved by GL-RM, we do not investigate if the spines are prevented from shrinking, or if they are de novo generated spines. This could be further explored in longitudinal studies, assessing the dynamic of the changes. Based on analysis of ketamine to overcome stress hormone-induced spine loss, a combination of restored spines and de novo spines was 
found, with some preference for de novo [111]. A similar combination approach may also be the case for GL-RM. This evidence that GL-RM has an effect at a pathophysiological level (not only a symptomatic level) is substantially valuable for translational efficacy within humans.

While this study has exciting new implications for selective GABAA targeting it is important to consider some of the study limitations. Electrophysiology results included a low sample size $(n=4)$ and therefore variability within samples can impact the results. At the beginning of potentiation there is increased variability and for this reason data should be viewed as an approximate continuum curve over concentrations versus an all-or-nothing potentiation response at each concentration. Future investigations in rodent should also include blood collection for plasma levels to confirm the circulating levels of compound in behaving animals, in order to map the behavioral outcomes to compound levels in the circulating system, or ideally directly in the brain. This would also help further characterize the therapeutic range and therapeutic window that has the most relevant and beneficial effects. Another limitation from this study can be found regarding the fact that we only used one behavioral assay to assess cognition, more specifically working memory. This is due to technical constraint, and to time commitment for other cognitive tasks that can be time consuming and would require days to weeks (sometimes even months) to train and assess cognitive functions in mice. Such design presents great limitations, in particular in the context of chronic drug treatment, which would require large amount of drug to sustain chronic administration. Future considerations should then include a broader battery of cognitive tests to build on these findings and eventually tease out efficacy in other cognitive domains such as short term and long term memory, recognition memory, cognitive flexibility and learning. Finally, while GL-RM has promising effects it would be interesting and worthwhile to resolve the two enantiomers and further study their individual therapeutic potential.

In conclusion, the therapeutic relevance and potential of a targeted PAM approach at GABA A receptors is reinforced. Building on previous studies, GL-II-73 continues to be a qualified candidate for improving cognition in various preclinical models. The addition of GL-I-54 to GL-II-73 as a racemic mixture remains relevant for cognitive performance and emotionality. Therefore, the present study reinforces the importance of selectively targeting GABAA-R subunits, namely $\alpha 5, \alpha 3$ and $\alpha 2$ to evoke specific therapeutic outcomes and how this may eventually address heterogeneous symptom profiles on multiple levels for disorders such as MDD.

\section{Funding and Disclosure}


This study was funded by CAMH Internal Fund (Discovery Fund). TP was awarded a Discovery Fund Fellowship at the time of the study execution.

\section{Acknowledgements}

The authors thank Mehrab Ali and Netta Ussyshkin for their support with administrative tasks along the way of the performance of the study. They also thank the staff of camh animal facility for the care provided to our animals over the course of the study. The authors also thank the different members from Charles River Laboratories and NeuroDigitech for their contribution on the data generation.

\section{Author Contributions}

TP and ES designed the study. YM, ZAK, DS and JMC synthesized the compounds tested in this study. TP and PL performed behavioral piece. Electrophysiology was outsourced to Charles River Laboratories. TP, $A B$ and $M M$ analyzed the data. TP and $A B$ wrote the manuscript. 


\section{References}

1. Disease, G.B.D., I. Injury, and C. Prevalence, Global, regional, and national incidence, prevalence, and years lived with disability for 328 diseases and injuries for 195 countries, 1990-2016: a systematic analysis for the Global Burden of Disease Study 2016. Lancet, 2017. 390(10100): p. 1211-1259.

2. MacQueen, G.M. and K.A. Memedovich, Cognitive dysfunction in major depression and bipolar disorder: Assessment and treatment options. Psychiatry and Clinical Neurosciences, 2017. 71(1): p. 18-27.

3. Malhi, G.S. and J.J. Mann, Depression. The Lancet, 2018. 392(10161): p. 2299-2312.

4. Gartlehner, G., et al., Pharmacological and non-pharmacological treatments for major depressive disorder: review of systematic reviews. BMJ Open, 2017. 7(6): p. e014912.

5. Hirschfeld, R.M.A., History and evolution of the monoamine hypothesis of depression. The Journal of Clinical Psychiatry, 2000. 61(Suppl6): p. 4-6.

6. Fogaca, M.V. and R.S. Duman, Cortical GABAergic Dysfunction in Stress and Depression: New Insights for Therapeutic Interventions. Front Cell Neurosci, 2019. 13: p. 87.

7. Chávez-Castillo, M., et al., Depression as a Neuroendocrine Disorder: Emerging Neuropsychopharmacological Approaches beyond Monoamines. Advances in Pharmacological Sciences, 2019. 2019: p. 7943481.

8. Duman, C.H. and R.S. Duman, Spine synapse remodeling in the pathophysiology and treatment of depression. Neurosci Lett, 2015. 601: p. 20-9.

9. Conradi, H.J., J. Ormel, and P. De Jonge, Presence of individual (residual) symptoms during depressive episodes and periods of remission: a 3-year prospective study. Psychological Medicine, 2011. 41(6): p. 1165-1174.

10. Fossati, P., A.M. Ergis, and J.F. Allilaire, [Executive functioning in unipolar depression: a review]. Encephale, 2002. 28(2): p. 97-107.

11. Bortolato, B., et al., Cognitive remission: a novel objective for the treatment of major depression? BMC Med, 2016. 14: p. 9.

12. Lee, R.S.C., et al., A meta-analysis of cognitive deficits in first-episode Major Depressive Disorder. Journal of Affective Disorders, 2012. 140(2): p. 113-124.

13. Majer, M., et al., Impaired divided attention predicts delayed response and risk to relapse in subjects with depressive disorders. Psychological Medicine, 2004. 34(8): p. 1453-1463.

14. Kang, H.J., et al., Decreased expression of synapse-related genes and loss of synapses in major depressive disorder. Nat Med, 2012. 18(9): p. 1413-7.

15. Holmes, S.E., et al., Lower synaptic density is associated with depression severity and network alterations. Nature Communications, 2019. 10(1): p. 1529.

16. Kang, H.J., et al., Decreased expression of synapse-related genes and loss of synapses in major depressive disorder. Nature Medicine, 2012. 18(9): p. 1413-1417.

17. Feyissa, A.M., et al., Reduced levels of NR2A and NR2B subunits of NMDA receptor and PSD-95 in the prefrontal cortex in major depression. Progress in Neuro-Psychopharmacology and Biological Psychiatry, 2009. 33(1): p. 70-75.

18. Christoffel, D.J., S.A. Golden, and S.J. Russo, Structural and synaptic plasticity in stress-related disorders. Reviews in the neurosciences, 2011. 22(5): p. 535-549.

19. Izquierdo, A., C.L. Wellman, and A. Holmes, Brief uncontrollable stress causes dendritic retraction in infralimbic cortex and resistance to fear extinction in mice. J Neurosci, 2006. 26(21): p. 5733-8.

20. Liston, C., et al., Stress-induced alterations in prefrontal cortical dendritic morphology predict selective impairments in perceptual attentional set-shifting. J Neurosci, 2006. 26(30): p. 7870-4. 
21. Shansky, R.M., et al., Stress-Induced Dendritic Remodeling in the Prefrontal Cortex is Circuit Specific. Cerebral Cortex, 2009. 19(10): p. 2479-2484.

22. Qiao, H., et al., Dendritic Spines in Depression: What We Learned from Animal Models. Neural plasticity, 2016. 2016: p. 8056370-8056370.

23. Phoumthipphavong, V., et al., Longitudinal Effects of Ketamine on Dendritic Architecture In Vivo in the Mouse Medial Frontal Cortex. eNeuro, 2016. 3(2): p. ENEURO.0133-15.2016.

24. Gerhard, D.M., et al., GABA interneurons are the cellular trigger for ketamine's rapid antidepressant actions. J Clin Invest, 2020. 130(3): p. 1336-1349.

25. Newton, D.F., et al., Chapter 28 - Altered GABAergic Function, Cortical Microcircuitry, and Information Processing in Depression, in Neurobiology of Depression, J. Quevedo, A.F. Carvalho, and C.A. Zarate, Editors. 2019, Academic Press. p. 315-329.

26. Berrettini, W.H., et al., Reduced plasma and CSF gamma-aminobutyric acid in affective illness: effect of lithium carbonate. Biological psychiatry, 1983. 18(2): p. 185-194.

27. Petty, F., et al., Plasma GABA in mood disorders. Psychopharmacology bulletin, 1990. 26(2): p. 157-61.

28. Petty, F., et al., Stability of plasma GABA at four-year follow-up in patients with primary unipolar depression. Biological psychiatry, 1995. 37(11): p. 806-810.

29. Petty, F., et al., Low plasma $\mathrm{\gamma}$-aminobutyric acid levels in male patients with depression. Biological Psychiatry, 1992. 32(4): p. 354-363.

30. Petty, F. and A.D. Sherman, Plasma GABA in psychiatric illness. J Affect Disorder, 1984. 6: p. 131138.

31. Petty, F., et al., Low plasma GABA is a trait-like marker for bipolar illness. Neuropsychopharmacology, 1993. 9(2): p. 125-32.

32. Bhagwagar, Z., et al., Low GABA concentrations in occipital cortex and anterior cingulate cortex in medication-free, recovered depressed patients. The international journal of neuropsychopharmacology / official scientific journal of the Collegium Internationale Neuropsychopharmacologicum (CINP), 2008. 11(2): p. 255-60.

33. Goddard, A.W., et al., Reductions in Occipital Cortex GABA Levels in Panic Disorder Detected With 1H-Magnetic Resonance Spectroscopy. Archives of General Psychiatry, 2001. 58(6): p. 556556.

34. Price, R.B., et al., Amino acid neurotransmitters assessed by proton magnetic resonance spectroscopy: relationship to treatment resistance in major depressive disorder. Biol Psychiatry, 2009. 65(9): p. 792-800.

35. Sanacora, G., et al., Subtype-specific alterations of gamma-aminobutyric acid and glutamate in patients with major depression. Arch Gen Psychiatry, 2004. 61(7): p. 705-13.

36. Sanacora, G., et al., Increased occipital cortex GABA concentrations in depressed patients after therapy with selective serotonin reuptake inhibitors. Am J Psychiatry, 2002. 159(4): p. 663-5.

37. Sanacora, G., et al., Subtype-specific alterations of gamma-aminobutyric acid and glutamate in patients with major depression. Archives of general psychiatry, 2004. 61(7): p. 705-13.

38. Sanacora, G., et al., Reduced Cortical -Aminobutyric Acid Levels in Depressed Patients Determined by Proton Magnetic Resonance Spectroscopy. Archives of General Psychiatry, 1999. 56(11): p. 1043-1043.

39. Hasler, G., et al., Reduced prefrontal glutamate/glutamine and gamma-aminobutyric acid levels in major depression determined using proton magnetic resonance spectroscopy. Archives of general psychiatry, 2007. 64(2): p. 193-200.

40. Gabbay, V., et al., Anterior Cingulate Cortexy-Aminobutyric Acid in Depressed Adolescents. Archives of General Psychiatry, 2012. 69(2): p. 139-139. 
41. Plante, D.T., et al., Reduced gamma-aminobutyric acid in occipital and anterior cingulate cortices in primary insomnia: a link to major depressive disorder? Neuropsychopharmacology, 2012. 37(6): p. 1548-57.

42. Mohler, H., GABA(A) receptor diversity and pharmacology. Cell Tissue Res, 2006. 326(2): p. 50516.

43. Sigel, E. and M. Ernst, The Benzodiazepine Binding Sites of GABAA Receptors. Trends in Pharmacological Sciences, 2018. 39(7): p. 659-671.

44. Pariente, A., et al., The Benzodiazepine-Dementia Disorders Link: Current State of Knowledge. CNS Drugs, 2016. 30(1): p. 1-7.

45. Rudolph, U., et al., Benzodiazepine actions mediated by specific gamma-aminobutyric acid(A) receptor subtypes. Nature, 1999. 401(6755): p. 796-800.

46. Atack, J.R., GABAA receptor alpha2/alpha3 subtype-selective modulators as potential nonsedating anxiolytics. Curr Top Behav Neurosci, 2010. 2: p. 331-60.

47. Engin, E., J. Liu, and U. Rudolph, a2-containing $G A B A(A)$ receptors: a target for the development of novel treatment strategies for CNS disorders. Pharmacology \& therapeutics, 2012. 136(2): $\mathrm{p}$. 142-152.

48. Koh, M.T., S. Rosenzweig-Lipson, and M. Gallagher, Selective GABA(A) alpha5 positive allosteric modulators improve cognitive function in aged rats with memory impairment. Neuropharmacology, 2013. 64: p. 145-52.

49. Prevot, T.D., et al., Novel Benzodiazepine-Like Ligands with Various Anxiolytic, Antidepressant, or Pro-Cognitive Profiles. Mol Neuropsychiatry, 2019. 5(2): p. 84-97.

50. Fritschy, J.M. and H. Mohler, GABAA-receptor heterogeneity in the adult rat brain: differential regional and cellular distribution of seven major subunits. J Comp Neurol, 1995. 359(1): p. 15494.

51. Sigurdsson, T. and S. Duvarci, Hippocampal-Prefrontal Interactions in Cognition, Behavior and Psychiatric Disease. Frontiers in systems neuroscience, 2016. 9: p. 190-190.

52. Koh, M.T., S. Rosenzweig-Lipson, and M. Gallagher, Selective GABA(A) $\alpha 5$ positive allosteric modulators improve cognitive function in aged rats with memory impairment. Neuropharmacology, 2013. 64(1): p. 145-152.

53. Prevot, T.D., et al., Reversal of Age-Related Neuronal Atrophy by $\alpha 5$-GABAA Receptor Positive Allosteric Modulation. Cerebral Cortex, 2020.

54. Krishnan, V. and E.J. Nestler, Animal models of depression: molecular perspectives. Current topics in behavioral neurosciences, 2011. 7: p. 121-147.

55. Planchez, B., A. Surget, and C. Belzung, Animal models of major depression: drawbacks and challenges. Journal of Neural Transmission, 2019. 126(11): p. 1383-1408.

56. Frisbee, J.C., et al., An Unpredictable Chronic Mild Stress Protocol for Instigating Depressive Symptoms, Behavioral Changes and Negative Health Outcomes in Rodents. Journal of visualized experiments : JoVE, 2015(106): p. 53109.

57. Antoniuk, S., et al., Chronic unpredictable mild stress for modeling depression in rodents: Metaanalysis of model reliability. Neuroscience \& Biobehavioral Reviews, 2019. 99: p. 101-116.

58. Shepard, R., C.E. Page, and L. Coutellier, Sensitivity of the prefrontal GABAergic system to chronic stress in male and female mice: Relevance for sex differences in stress-related disorders. Neuroscience, 2016. 332: p. 1-12.

59. Ma, K., et al., Impaired GABA synthesis, uptake and release are associated with depression-like behaviors induced by chronic mild stress. Transl Psychiatry, 2016. 6(10): p. e910.

60. Luscher, B., Q. Shen, and N. Sahir, The GABAergic deficit hypothesis of major depressive disorder. Mol Psychiatry, 2011. 16(4): p. 383-406. 
61. Karolewicz, B., et al., Reduced level of glutamic acid decarboxylase-67 kDa in the prefrontal cortex in major depression. Int J Neuropsychopharmacol, 2010. 13(4): p. 411-20.

62. Romeo, B., et al., Meta-analysis of central and peripheral 1 -aminobutyric acid levels in patients with unipolar and bipolar depression. J Psychiatry Neurosci, 2018. 43(1): p. 58-66.

63. Hasler, G., et al., Reduced prefrontal glutamate/glutamine and gamma-aminobutyric acid levels in major depression determined using proton magnetic resonance spectroscopy. Arch Gen Psychiatry, 2007. 64(2): p. 193-200.

64. Banasr, M., et al., Characterization of GABAergic marker expression in the chronic unpredictable stress model of depression. Chronic Stress (Thousand Oaks), 2017. 1.

65. Maluach, A.M., et al., Increased Neuronal DNA/RNA Oxidation in the Frontal Cortex of Mice Subjected to Unpredictable Chronic Mild Stress. Chronic Stress (Thousand Oaks), 2017. 1.

66. Cryan, J.F. and K. Kaupmann, Don't worry 'B' happy!: a role for $G A B A(B)$ receptors in anxiety and depression. Trends Pharmacol Sci, 2005. 26(1): p. 36-43.

67. Fogaça, M.V. and R.S. Duman, Cortical GABAergic Dysfunction in Stress and Depression: New Insights for Therapeutic Interventions. Frontiers in cellular neuroscience, 2019. 13: p. 87-87.

68. Czéh, B., et al., Long-Term Stress Disrupts the Structural and Functional Integrity of GABAergic Neuronal Networks in the Medial Prefrontal Cortex of Rats. Front Cell Neurosci, 2018. 12: p. 148.

69. Fee, C., M. Banasr, and E. Sibille, Somatostatin-Positive Gamma-Aminobutyric Acid Interneuron Deficits in Depression: Cortical Microcircuit and Therapeutic Perspectives. Biol Psychiatry, 2017. 82(8): p. 549-559.

70. Billakota, S., et al., Personalized medicine: Vinpocetine to reverse effects of GABRB3 mutation. Epilepsia, 2019. 60(12): p. 2459-2465.

71. Kirsch, G.E., et al., Electrophysiology-Based Assays to Detect Subtype-Selective Modulation of Human Nicotinic Acetylcholine Receptors. ASSAY and Drug Development Technologies, 2016. 14(6): p. 333-344.

72. Di, L., et al., Optimization of a higher throughput microsomal stability screening assay for profiling drug discovery candidates. Journal of biomolecular screening, 2003. 8(4): p. 453-462.

73. Nollet, M., A.M. Le Guisquet, and C. Belzung, Models of depression: unpredictable chronic mild stress in mice. Curr Protoc Pharmacol, 2013. Chapter 5: p. Unit 5.65.

74. Prevot, T.D., et al., Residual avoidance: $A$ new, consistent and repeatable readout of chronic stress-induced conflict anxiety reversible by antidepressant treatment. Neuropharmacology, 2019. 153: p. 98-110.

75. Faucher, P., et al., Hippocampal Injections of Oligomeric Amyloid B-peptide (1-42) Induce Selective Working Memory Deficits and Long-lasting Alterations of ERK Signaling Pathway. Front Aging Neurosci, 2015. 7: p. 245.

76. Wu, C.C., et al., Selective vulnerability of dentate granule cells prior to amyloid deposition in PDAPP mice: digital morphometric analyses. Proc Natl Acad Sci U S A, 2004. 101(18): p. 7141-6.

77. Fee, C., et al., Behavioral deficits induced by somatostatin-positive GABA neuron silencing are rescued by alpha 5 GABA-A receptor potentiation. Int J Neuropsychopharmacol, 2021.

78. Prevot, T.D., et al., Reversal of Age-Related Neuronal Atrophy by alpha5-GABAA Receptor Positive Allosteric Modulation. Cereb Cortex, 2021. 31(2): p. 1395-1408.

79. Geracitano, R., et al., Functional expression of the GABA(A) receptor $\alpha 2$ and $\alpha 3$ subunits at synapses between intercalated medial paracapsular neurons of mouse amygdala. Front Neural Circuits, 2012. 6: p. 32.

80. Stefanits, H., et al., GABAA receptor subunits in the human amygdala and hippocampus: Immunohistochemical distribution of 7 subunits. Journal of Comparative Neurology, 2018. 526(2): p. 324-348. 
81. Marowsky, A., J.M. Fritschy, and K.E. Vogt, Functional mapping of GABA A receptor subtypes in the amygdala. Eur J Neurosci, 2004. 20(5): p. 1281-9.

82. Heldt, S.A. and K.J. Ressler, Training-induced changes in the expression of GABAA-associated genes in the amygdala after the acquisition and extinction of Pavlovian fear. Eur J Neurosci, 2007. 26(12): p. 3631-44.

83. Dias, R., et al., Evidence for a significant role of alpha 3-containing GABAA receptors in mediating the anxiolytic effects of benzodiazepines. J Neurosci, 2005. 25(46): p. 10682-8.

84. Möhler, H., The GABA system in anxiety and depression and its therapeutic potential. Neuropharmacology, 2012. 62(1): p. 42-53.

85. Collins, I., et al., 3-Heteroaryl-2-pyridones: benzodiazepine site ligands with functional delectivity for alpha 2/alpha 3-subtypes of human GABA(A) receptor-ion channels. J Med Chem, 2002. 45(9): p. 1887-900.

86. Prevot, T. and E. Sibille, Altered GABA-mediated information processing and cognitive dysfunctions in depression and other brain disorders. Mol Psychiatry, 2021. 26(1): p. 151-167.

87. Wang, J., et al., Weak-binding molecules are not drugs?-toward a systematic strategy for finding effective weak-binding drugs. Brief Bioinform, 2017. 18(2): p. 321-332.

88. McKernan, R.M., et al., Sedative but not anxiolytic properties of benzodiazepines are mediated by the GABAA receptor $\alpha 1$ subtype. Nature Neuroscience, 2000. 3(6): p. 587-592.

89. Budău, M., et al., Chirality of Modern Antidepressants: An Overview. Adv Pharm Bull, 2017. 7(4): p. $495-500$.

90. Sekhon, B.S., Exploiting the power of stereochemistry in drugs: an overview of racemic and enantiopure drugs. J. Mod. Med. Chem, 2013. 1: p. 10-36.

91. Nguyen, L.A., H. He, and C. Pham-Huy, Chiral drugs: an overview. International journal of biomedical science : IJBS, 2006. 2(2): p. 85-100.

92. Perez-Caballero, L., et al., Fluoxetine: a case history of its discovery and preclinical development. Expert Opin Drug Discov, 2014. 9(5): p. 567-78.

93. Bezchlibnyk-Butler, K., I. Aleksic, and S.H. Kennedy, Citalopram--a review of pharmacological and clinical effects. J Psychiatry Neurosci, 2000. 25(3): p. 241-54.

94. Fuller, R.W., et al., Comparison of norfluoxetine enantiomers as serotonin uptake inhibitors in vivo. Neuropharmacology, 1992. 31(10): p. 997-1000.

95. Mineur, Y.S., C. Belzung, and W.E. Crusio, Effects of unpredictable chronic mild stress on anxiety and depression-like behavior in mice. Behav Brain Res, 2006. 175(1): p. 43-50.

96. Aarts, E., et al., The light spot test: Measuring anxiety in mice in an automated home-cage environment. Behav Brain Res, 2015. 294: p. 123-30.

97. Gilabert-Juan, J., et al., Chronic stress alters inhibitory networks in the medial prefrontal cortex of adult mice. Brain Struct Funct, 2013. 218(6): p. 1591-605.

98. Lin, L.C. and E. Sibille, Somatostatin, neuronal vulnerability and behavioral emotionality. Mol Psychiatry, 2015. 20(3): p. 377-87.

99. Prévot, T. and E. Sibille, Altered GABA-mediated information processing and cognitive dysfunctions in depression and other brain disorders. Mol Psychiatry, 2020.

100. Abbas, A.I., et al., Somatostatin Interneurons Facilitate Hippocampal-Prefrontal Synchrony and Prefrontal Spatial Encoding. Neuron, 2018. 100(4): p. 926-939.e3.

101. Prévôt, T.D., et al., sst(2)-receptor gene deletion exacerbates chronic stress-induced deficits: Consequences for emotional and cognitive ageing. Prog Neuropsychopharmacol Biol Psychiatry, 2018. 86: p. 390-400.

102. Gumuslu, E., et al., The Antidepressant Agomelatine Improves Memory Deterioration and Upregulates CREB and BDNF Gene Expression Levels in Unpredictable Chronic Mild Stress (UCMS)-Exposed Mice. Drug Target Insights, 2014. 8: p. 11-21. 
103. Fee, C., et al., Chronic Stress-induced Behaviors Correlate with Exacerbated Acute Stress-induced Cingulate Cortex and Ventral Hippocampus Activation. Neuroscience, 2020. 440: p. 113-129.

104. Stefanits, H., et al., GABA(A) receptor subunits in the human amygdala and hippocampus: Immunohistochemical distribution of 7 subunits. J Comp Neurol, 2018. 526(2): p. 324-348.

105. Hörtnagl, H., et al., Patterns of $m R N A$ and protein expression for 12 GABAA receptor subunits in the mouse brain. Neuroscience, 2013. 236: p. 345-372.

106. Duveau, V., et al., Spatiotemporal specificity of GABAA receptor-mediated regulation of adult hippocampal neurogenesis. Eur J Neurosci, 2011. 34(3): p. 362-73.

107. Deprez, F., et al., Partial inactivation of GABAA receptors containing the $\alpha 5$ subunit affects the development of adult-born dentate gyrus granule cells. Eur J Neurosci, 2016. 44(5): p. 2258-71.

108. Brady, M.L. and T.C. Jacob, Synaptic localization of $\alpha 5$ GABA (A) receptors via gephyrin interaction regulates dendritic outgrowth and spine maturation. Developmental Neurobiology, 2015. 75(11): p. 1241-1251.

109. Schulz, J.M., et al., Dendrite-targeting interneurons control synaptic NMDA-receptor activation via nonlinear $\alpha 5-G A B A(A)$ receptors. Nat Commun, 2018. 9(1): p. 3576.

110. Serwanski, D.R., et al., Synaptic and nonsynaptic localization of GABAA receptors containing the alpha5 subunit in the rat brain. J Comp Neurol, 2006. 499(3): p. 458-70.

111. Moda-Sava, R.N., et al., Sustained rescue of prefrontal circuit dysfunction by antidepressantinduced spine formation. Science, 2019. 364(6436). 


\section{Figure Legend}

\section{Figure 1. Electrophysiological and behavioral profiles of GL-II-73 and GL-I-54}

Electrophysiological recordings were obtained from HEK-293T cells transfected with full-length cDNA for human GABAA receptor subtypes $\alpha 1 \beta 3 \gamma 2, \alpha 2 \beta 3 \gamma 2, \alpha 3 \beta 3 \gamma 2, \alpha 4 \beta 3 \gamma 2$ or $\alpha 5 \beta 3 \gamma 2$, in presence of GL-II-73 (A) or GL-I-54 (B), and in presence of GABA in the medium (5 $\mu \mathrm{M})$. GL-II-73 potentiation of $\alpha 5-G A B A A-R$ was significantly higher than $\alpha 1, \alpha 2$ and $\alpha 3$ at concentration higher than $3.3 \mu \mathrm{M}(\mathrm{A})$. GL-I-54 potentiation of $\alpha 3-G A B A A-R$ was significantly higher than $\alpha 1, \alpha 2$ and $\alpha 5$ at concentration higher than $3.3 \mu \mathrm{M}$ (B). GL-I-54 was further tested in the elevated plus maze at the dose of $10 \mathrm{mg} / \mathrm{kg}$. Percent time spent in the open arms (C) and number of entries in the open arms (D) showed significant increase in animals receiving GL-I-54, compared to vehicle. Finally, GL-I-54 was tested in the $\mathrm{Y}$ maze task, assessing working memory. To induce a working memory deficit mice were subjected to 7 days of chronic restraint stress. 30 min prior to testing, mice were injected with vehicle or 1,5 or $10 \mathrm{mg} / \mathrm{kg}$ of GL-I-54. Animals subjected to CRS and receiving vehicle showed a significant decrease in alternation rate, suggesting a working memory deficit. Animal subjected to CRS and receiving the highest dose of GL-I-54 showed significant increase in alternation rate, suggesting reversal of working memory deficits induced by CRS. ${ }^{*} p<0.05,{ }^{* *} p<0.01,{ }^{* * *} p<0.001$ compared to vehicle; $\$ p<0.05, \$ \$ p<0.01, \$ \$ \$ p<0.001$ compared to $\alpha 1$; ]p $<0.05, p<0.01, p<0.001$ compared $\alpha 2 ; \# p<0.05, \# \# p<0.01$, \#\#\#p<0.001 compared $\alpha 3, \phi p<0.05$, $\phi \phi \mathrm{p}<0.01, \phi \phi \phi \mathrm{p}<0.001$ compared to $\alpha 5$; $\mathrm{t}=$ trend.

Figure 2. Effect of acute treatment of GL-RM on anxiety, emotionality and working memory deficits in mice subjected to chronic stress

Male and female mice were tested at baseline in the YMaze, the phenotyper and the sucrose consumption test prior to being subjected to 6 weeks of UCMS (A). Weekly, mice were tested in the phenotyper test, the sucrose consumption and their weight and coat state were measured. After 6 weeks of UCMS, acute injections were performed 30 minutes prior to behavioral testing. In the elevated plus maze, time spent (B) and entries (C) in the open arms were measured. Statistical analyses revealed an effect of UCMS in the percent of entries in the open arms. In the novelty suppressed feeding test, latency to approach (D) and latency to bite (E) were assessed. Statistical analyses showed that acute GLRM treatment reduces latency to approach in mice subjected to UCMS. In the phenotyper test (F), mice were placed in the box overnight, where a stressful stimulus was applied at $11 \mathrm{pm}$, for $1 \mathrm{hr}$. Time spent in the shelter zone showed that mice subjected to UCMS spent more time in the shelter than control mice. 
Calculating a residual avoidance score $(G)$, statistical analyses showed a significant increase in residual avoidance in mice subjected to UCMS. In the sucrose consumption test (H), mice subjected to UCMS showed a significant decrease in preference to sucrose. In the forced swim test (I), mice subjected to UCMS showed increased immobility, while mice treated with GL-RM showed a reduction in immobility. Combining the individual score into a global z-score (J), statistical analyses confirmed a significant impact of UCMS, with reduced effect of GL-RM. Finally, mice were tested in the Y maze (K), where statistical analyses showed altered alternation with UCMS, which is reversed by acute GL-RM treatment. * $p<0.05$, ${ }^{* *} p<0.01, * * * p<0.001$ effect of UCMS, \#p<0.05, \#\#p<0.01, effect of GL-RM

Figure 3. Effect of chronic treatment of GL-RM on anxiety, emotionality and working memory deficits in mice subjected to chronic stress

Male and female mice were tested at baseline in the YMaze, the phenotyper and the sucrose consumption test prior to being subjected to 6 weeks of UCMS (A). After 3 weeks of UCMS, chronic treatment with GL-RM in the drinking water was initiated, for a total of 4 weeks. Weekly, mice were tested in the phenotyper test, the sucrose consumption and their weight and coat state were measured. After 6 weeks of UCMS, and 3 weeks of treatment, mice were tested in the elevated plus maze. Time spent (B) and entries (C) in the open arms were measured, but did not show statistical differences. Mice were also tested in the open field for the time spent (D) and number of entries (E) in the inner zone. Again, statistical analyses did not reveal any effect of UCMS nor treatment. In the novelty suppressed feeding test, latency to approach (F) and latency to bite $(G)$ were assessed. Mice were tested in the Phenotyper weekly, and the residual avoidance scores from week 3 to 6 were analyzed, since the treatment was onboard during these testing periods $(\mathrm{H})$. In the forced swim test (I), statistical analyses did not reveal significant differences between groups. In the sucrose consumption test (J), mice receiving chronic GL-RM showed a significant increase in preference to sucrose. Combining the individual score into a global z-score (J), statistical analyses confirmed a significant impact of UCMS at increasing emotionality, while chronic GL-RM reduced emotionality. Finally, mice were tested in the $Y$ maze $(K)$, where statistical analyses showed altered alternation with UCMS, which is reversed by acute GL-RM treatment. ${ }^{*} p<0.05,{ }^{* *} p<0.01, * * * p<0.001$ effect of UCMS, \#p<0.05, \#\#p<0.01, effect of GL-RM

Figure 4. Chronic treatment with GL-RM reverses chronic-stress induced spine density reduction in the PFC and the CA1.

After completion of the behavioral screening, mice were euthanized and brains were stained with GolgiCox solution. Pyramidal neurons ( $N=6$ per mouse) from 4 mice per group $(A-C)$ were analyzed for 
dendritic length, spine counts and spine density. Basal and apical spine densities were measured in the PFC (D) and the CA1 of the hippocampus (E). ANOVA in the basal and apical segments revealed significant differences between groups, in both brain regions. This difference was explained by a decrease in spine density in mice subjected to UCMS compared to Control mice that was partially reversed by chronic treatment with GL-RM. * $p<0.05$, ** $p<0.01,{ }^{* * *} p<0.001$ compared to "UCMS"; $+p<0.05,++p<0.01,+++p<0.001$ compared to "Control". Scale bar in (A-C) represents $50 \mu \mathrm{m}$.

Figure 5. Morphological changes in the PFC and CA1 correlates with behavioral outcomes.

Morphological features from the PFC and the CA1 were correlated to behavioral outcomes, mostly the alternation rate $(A-D)$ and the z-emotionality (E-F). Basal (A) and apical (B) spine density in the PFC correlated positively with the alternation rate, where higher density was associated with higher alternation rate, i.e. higher memory performances. Similar findings were reported when looking at basal and apical spine density from the CA1 (C-D). Basal and Apical dendritic length in the CA1 also correlated with z-emotionality, this time negatively (E-F), where higher dendritic length was associated with lower z-emotionality, i.e. lower anxiety- and depressive-like behaviors. 


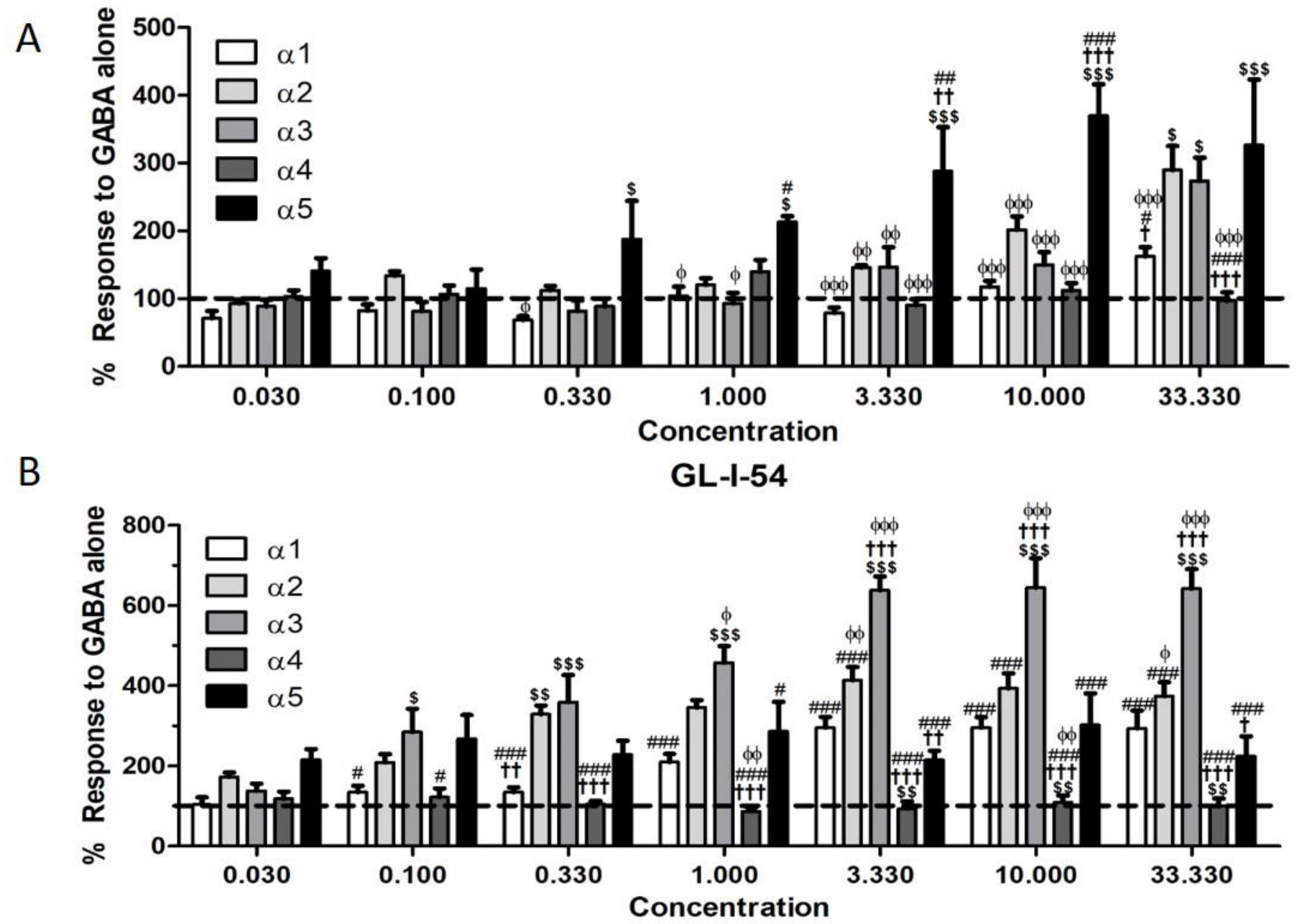

C

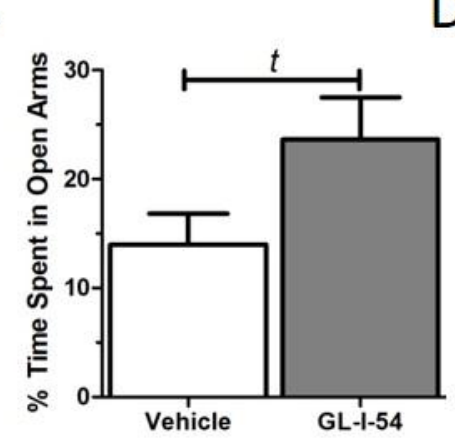

D

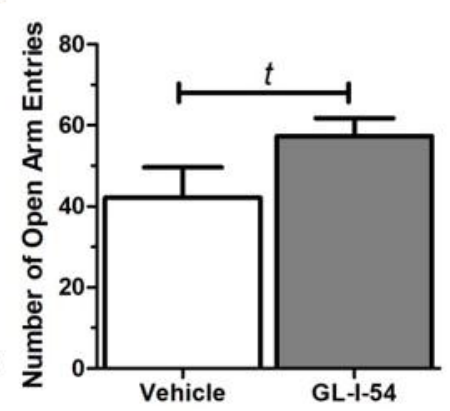

E

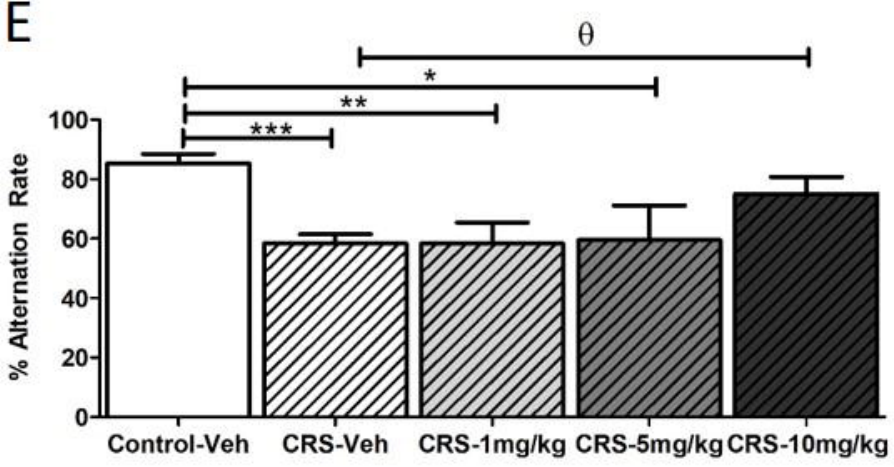


A

Week 0 (Baseline)

Y Maze

Coat State

Phenotyper

Sucrose Consumption
Week 1-6:

Coat State

Phenotyper

Sucrose Consumption
Week 7 Week 8

Y Maze FST

EPM

NSF

\begin{tabular}{|l|l|l|l|}
\hline Week 0 & Week 1-6 & Week 7 & Week 8 \\
\hline
\end{tabular}

Control / Vehicle

Control / GL-RM

UCMS / Vehicle

UCMS / GL-RM
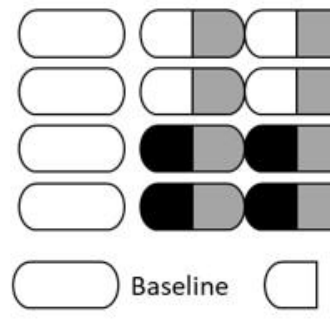

Week 1-6

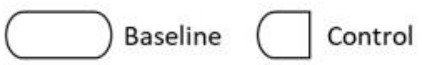

$\square$ UCMS $\square$ Vehicle

GL-RM
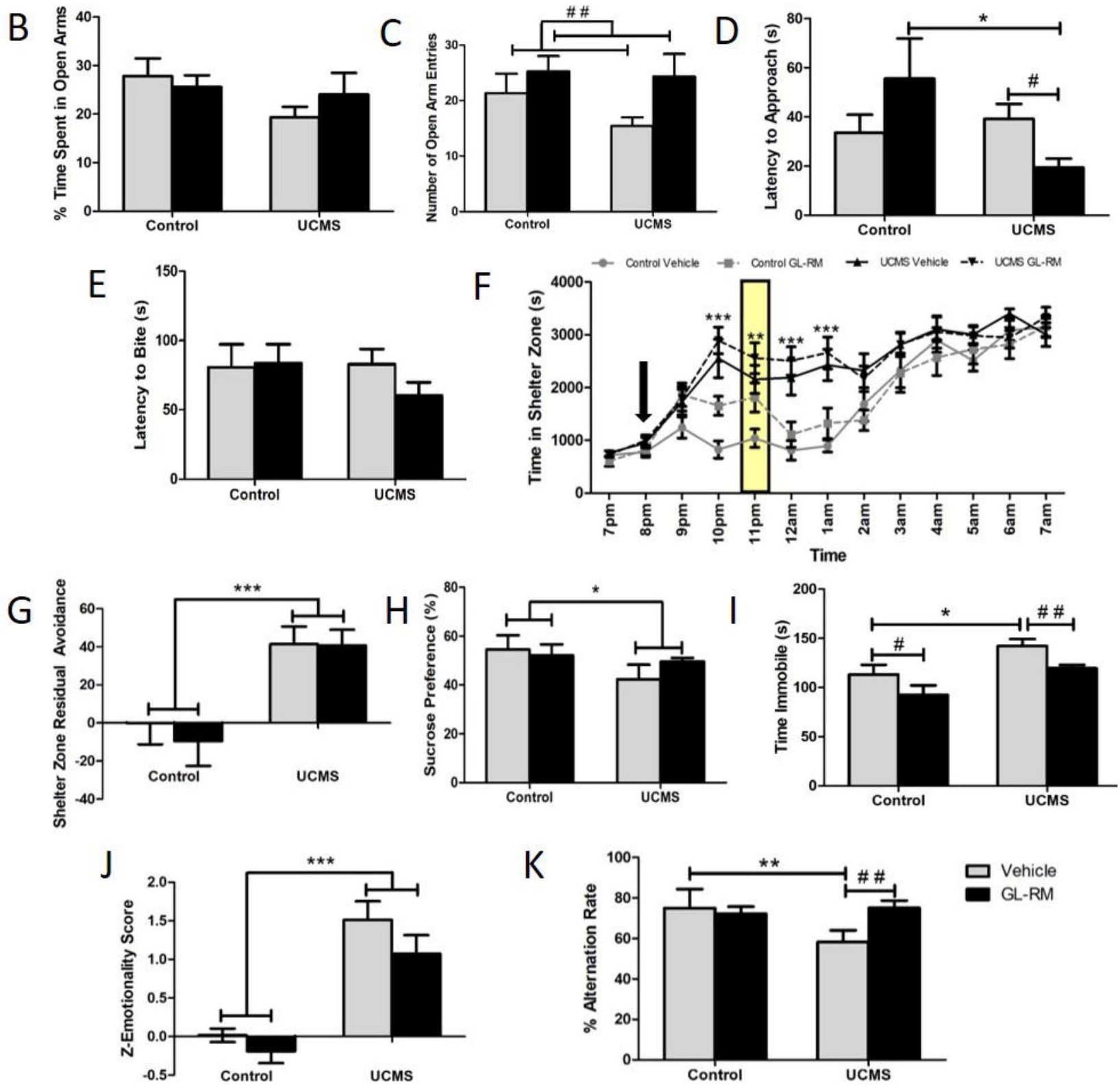

$\square$ Vehicle

GL-RM 


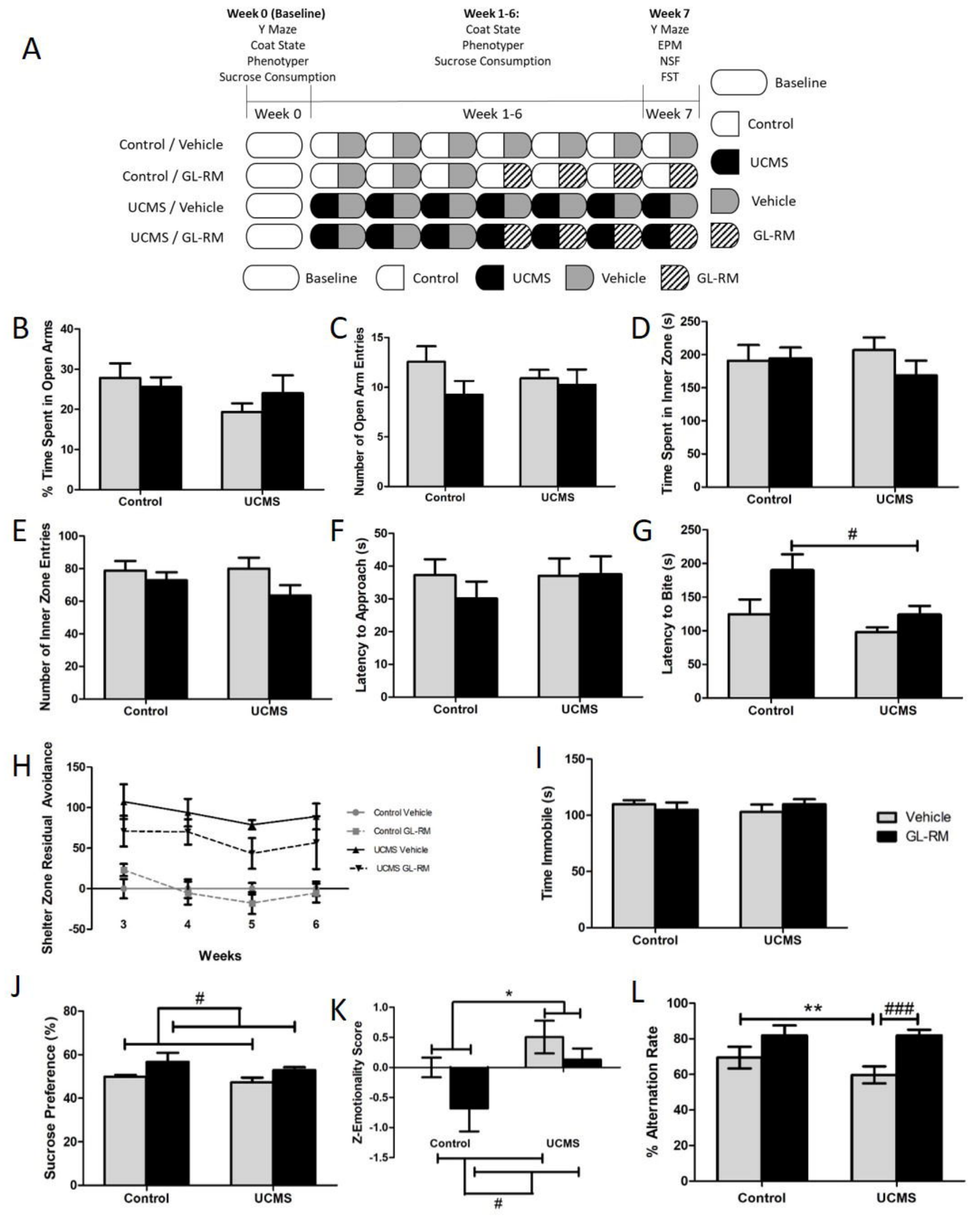




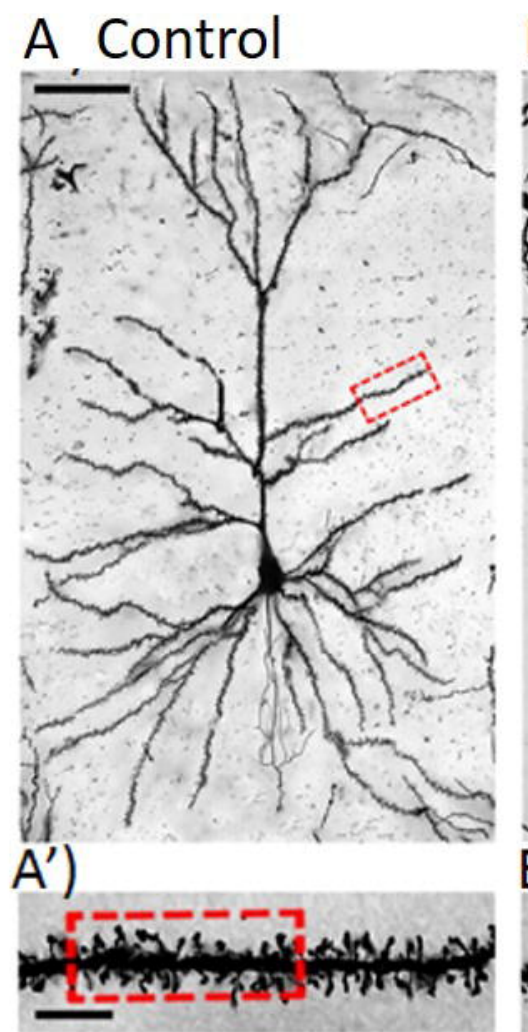

B UCMS

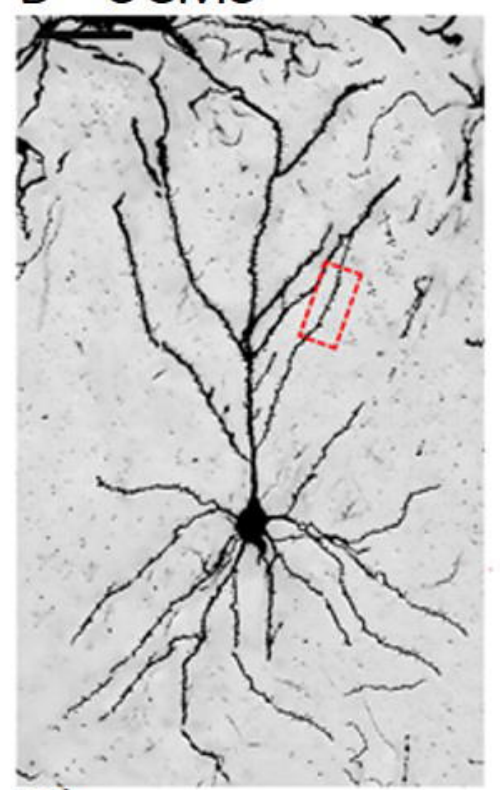

$\left.B^{\prime}\right)$

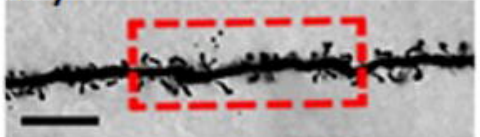

C UCMS+GL-RM

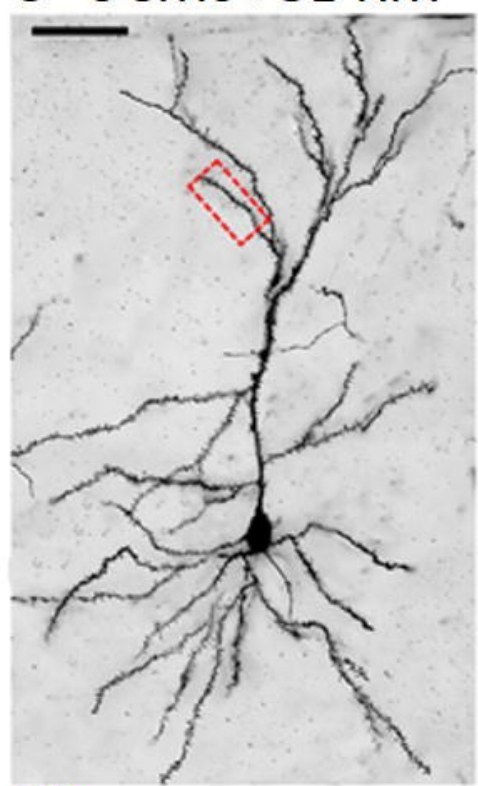

C')

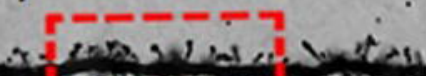

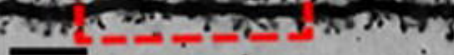

D

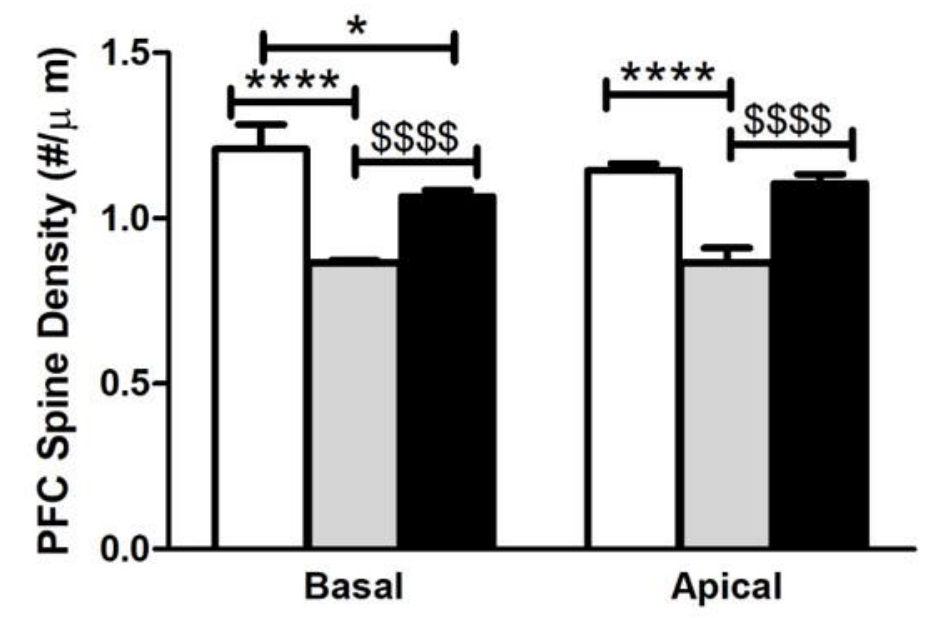

$\mathrm{E}$

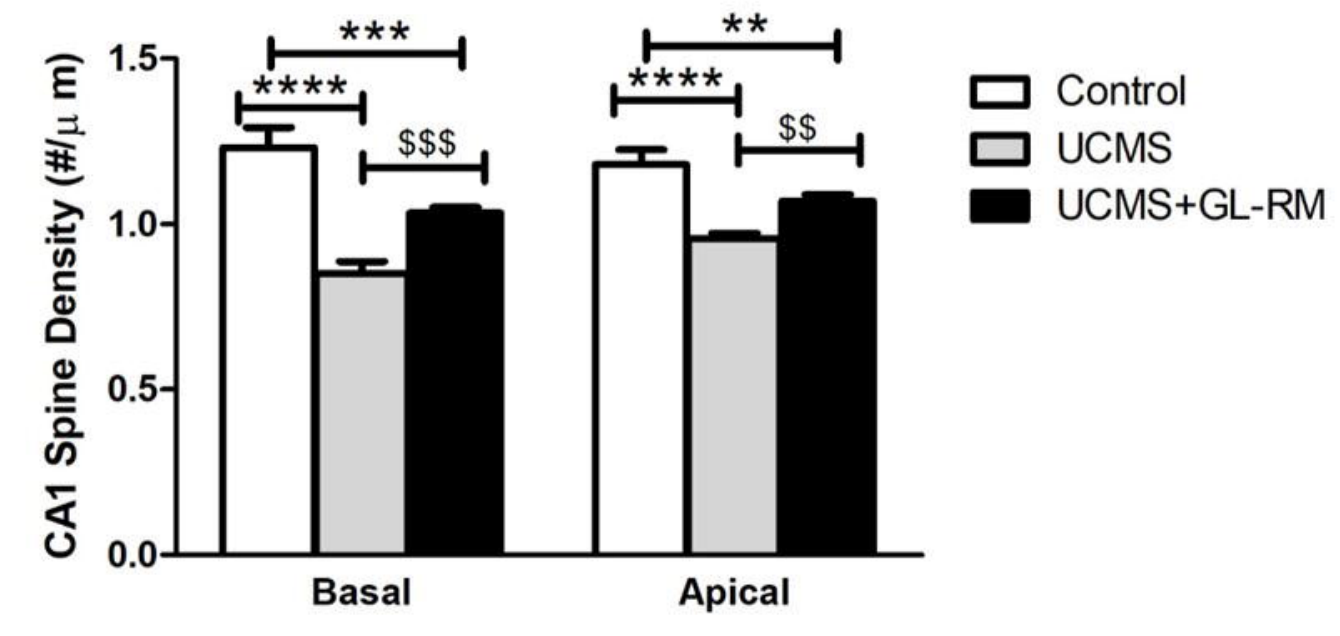

$\square$ Control

$\square$ UCMS

UCMS+GL-RM 


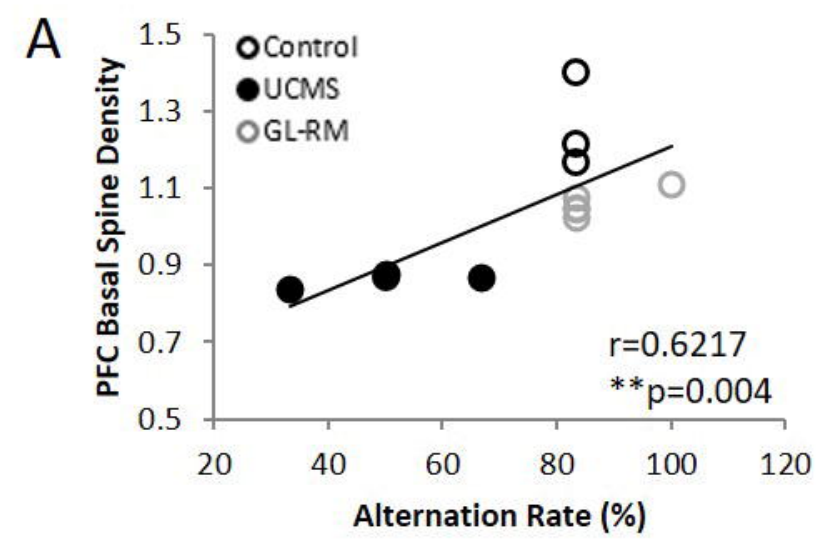

B

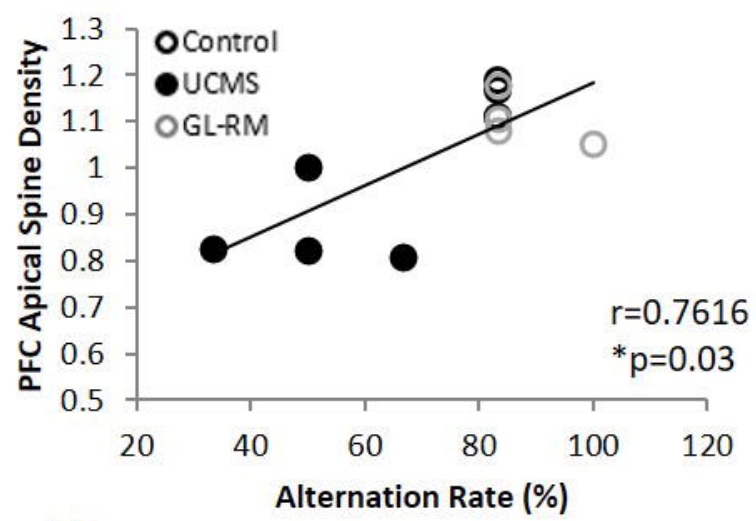

C
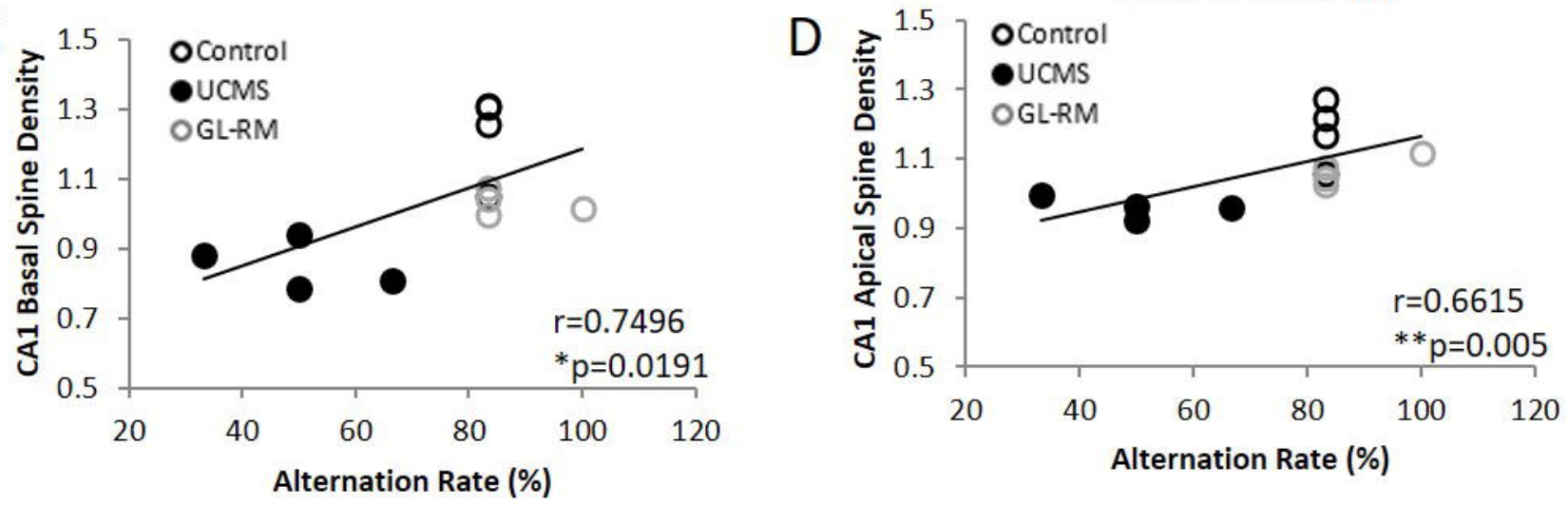

E

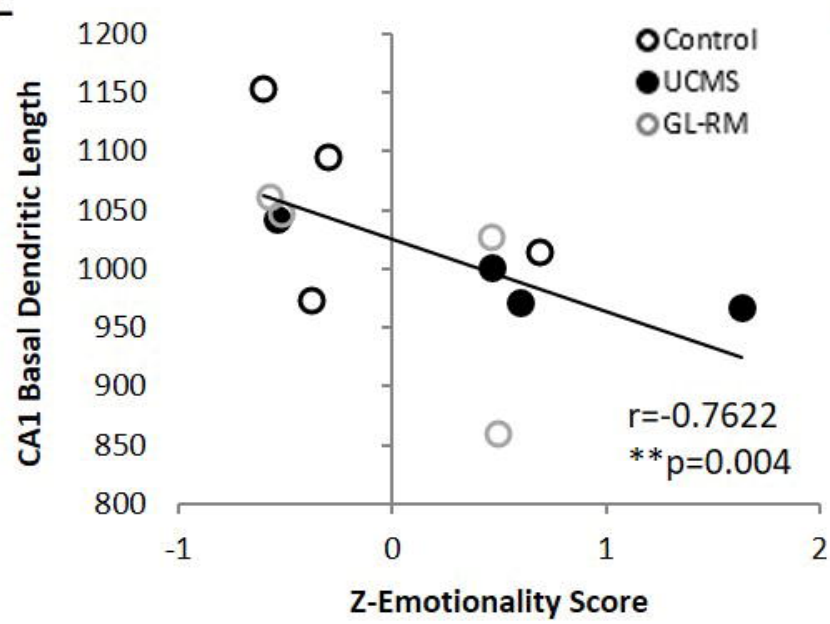

F

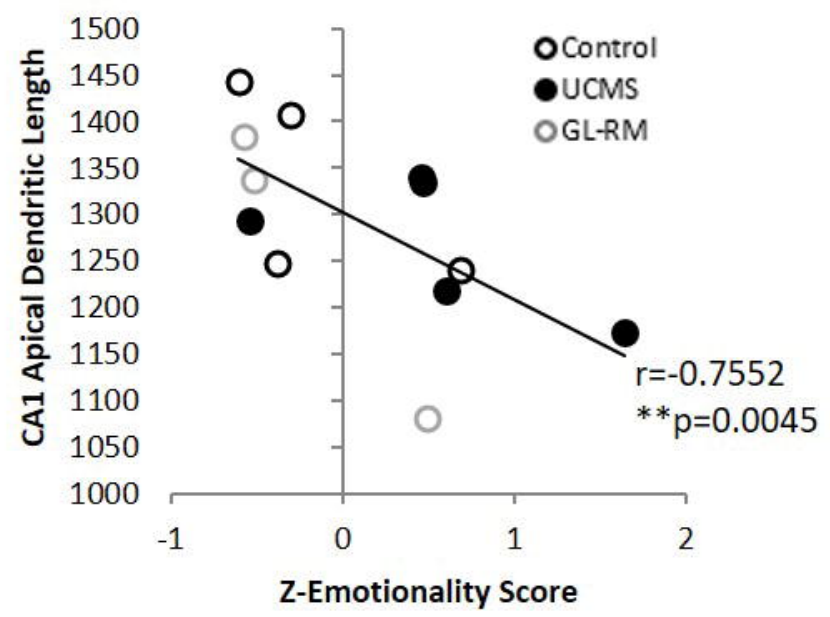

